\title{
Multivariate ANALysis of PePtide-Driven NuCleation AND GROWTH OF AU NANOPARTICLES
}

\author{
A PREPRINT
}

\author{
Kacper J. Lachowski ${ }^{1,2}$, Kiran Vaddi ${ }^{1}$, Nada Y. Naser ${ }^{1}$, François Baneyx ${ }^{1}$, Lilo D. Pozzo ${ }^{1,2,3}$
}

February 2022

而

\begin{abstract}
In this work we synthesize gold nanostructures using a liquid-handling robot and present new data analysis methods to quantitatively compare the effect of peptides and peptide modifications on gold nanoparticle synthesis outcomes. The peptides used were gold binding peptides Z2 and AG3, as well as five $\mathrm{Z} 2$ variants obtained by sequence modification or conjugation of a lipid tail. Four different concentrations of peptide, reducing agent (HEPES), and precursor $\left(\mathrm{HAuCl}_{4}\right)$ were used to synthesize 64 different reagent combinations for each of the seven peptides. Each sample was characterized using UV-Vis spectroscopy, which serves as a proxy for changes in nanoparticle structure, and enabled comparisons of how peptide modifications and reagent conditions affect synthesis outcomes. We then used functional data analysis to extract a pairwise signal correlation distance between each of the peptides. The signal correlation distance quantified how different the set of 64 spectra, and therefore the synthesis outcomes, was from one peptide to the other. We show that substitution of methionine for isoleucine in $\mathrm{Z} 2$ has a profound impact on synthesis outcomes when compared with conjugation of a lipid tail. Electron microscopy and ultra-small angle X-ray scattering were used to corroborate our conclusions from spectroscopy experiments by directly characterizing the structure of a smaller set of samples. Scanning electron microscopy revealed interconnected plate-like structures in samples prepared with a Z2 variant with all methionines substituted by isoleucine. Z2 peptides modified with a lipid tail on the other hand, formed nanoparticles which were more colloidally stable than those prepared with non-lipidated peptides, contained no plate-like particles, and whose size depended on the ratio of peptide to precursor.
\end{abstract}

\section{Introduction}

The structure and composition of inorganic colloidal materials enable several unique properties and functions. Structure encompasses the morphology, characteristic dimensions, polydispersity, and crystal structure of nanoscale materials. Control of thermodynamic and kinetic processes during synthesis is required to achieve desired structural features. Particles which are composed of metals (e.g. $\mathrm{Au}, \mathrm{Ag}, \mathrm{Pt}, \mathrm{Cu}$ ) and have nanoscale structures exhibit localized surface plasmon resonance (LSPR) in response to light [1]. LSPR has been used in chemical sensing, enhanced Raman spectroscopy, light harvesting, catalysis, medical imaging, and other applications [2]. The particle's structure is directly related to its LSPR response. Therefore, simple optical extinction measurements can provide information about particle structure within a sample. In the simplest case of a spherical nanoparticle, the magnitude and wavelength of the LSPR band is related to the diameter of the particle [1]. Different particle morphologies such as nanorods or nanotriangles allow for an additional mode of plasmonic resonance. Proximity to other particles can also modify the optical response through plasmonic coupling. As the size of features directly influences the optical properties of plasmonic particles, monodispersity is a desirable trait to obtain maximum control over the optical response. The crystal structure of metallic nanoparticles may be generally described as face-centered cubic (fcc), but the exact structural order at the surface can

\footnotetext{
${ }^{1}$ Department of Chemical Engineering, University of Washington, Seattle, Washington 98195, United States

${ }^{2}$ Molecular Engineering and Sciences Institute, University of Washington, Seattle, Washington 98195, United States

${ }^{3}$ Department of Materials Science and Engineering, University of Washington, Seattle, Washington 98195, United States
} 
vary depending on the method of synthesis. This is important because the order at the nanoparticle surface is related to its catalytic properties [3].

Efforts at improving control over nanoparticle structure and spatial organization have often taken inspiration from biomineralization. Specifically, researchers seek to emulate the mild synthesis conditions, use of molecules with molecular recognition, and structural complexity that nature has achieved. Sequence-defined molecules such as DNA, peptides, and peptoids are attractive choices for bio-inspired synthesis approaches and have been used to control the nucleation, growth, and self-assembly of inorganic materials [4, 5, 6]. Modifying the sequence of a molecule often has a significant impact on how the molecule interacts with inorganic precursors and particle surfaces [7, 8]. In turn, these modifications can lead to changes in nanoparticle formation mechanisms, colloidal stability, reaction kinetics, and ultimately the function of the synthesized material [3].

Research into understanding molecular design rules for peptide mediated Au nanoparticle research has continued to receive significant attention due to extensive potential applications for plasmonic nanoparticles. Au nanoparticle synthesis also serves as a good model system for method development. Following several discoveries of peptides with strong binding affinities to Au surfaces using biopanning methods, Tan et al.[9] performed a systematic study of each naturally occurring amino acid's contribution to Au binding and reduction. The authors synthesized peptides with each of the amino acids interdigitated with histidine. The interdigitated peptides were then used in experiments to assign scores for each amino acid's Au binding and reducing ability. However, one of the limitations of this work is related to one of the motivations for undertaking it in the first place - the order and identity of amino acids in the sequence can lead to surprising changes in peptide interaction with particle surfaces and precursors. Moreover, as was shown by Munro et al. [10], varying solution and reagent conditions will modify how a peptide performs in nanoparticle synthesis. Therefore, design rules obtained using a particular and narrow set of experimental conditions may be limited in their applicability to other experimental conditions. Munro et al. studied the effects of $\mathrm{pH}$, ionic strength, and ionic composition on the formation of Au nanoparticles using the peptide AuBP1. However, unlike AuBP1, not all peptides which have a strong binding affinity towards gold surfaces also have the appreciable capability of reducing Au(III), and therefore require the use of a reducing agent. Insights into molecular design rules can be generated by studying sequence rearrangement, amino acid substitutions or additions, and by comparison of known peptides to one another. One example of the latter is the work of Li et al., who compared the use of several Au binding peptides on the formation of Au nanoparticles at fixed molar concentrations of peptide, $\mathrm{HAuCl}_{4}$, and a reducing agent [11]. The researchers noted that each peptide's theoretical isoelectric point was a good predictor of zeta potential sign, but no peptide feature was identified as a good predictor of gold nanoparticle size or polydispersity. Finally, there are also several examples in which conjugation of a lipid tail to the peptide $\mathrm{N}$-terminus resulted in the peptide modifying the synthesis mechanism by acting as a template for nanoparticle growth and assembly [12, 13, 14, 15, 16, 17].

In this paper, we seek to address the lack of systematic experimental and data analysis methods for studying connections between peptide modifications (amino acid content, sequence order, lipid tail conjugation etc.) and synthesis outcomes within a large scope of experimental conditions. There has been a surge of interest and breakthroughs in applying high-throughput experimentation, design of experiments, and new data analysis methods to accelerate colloidal synthesis research. Often, the pursued objective is an optimization of a particular nanoparticle structure or characterization of phases in the experimental design space [18, 19]. In contrast, our work is concerned with using high-throughput experimentation and data analysis to understand the role of peptide modifications on Au nanoparticle synthesis. We demonstrated our approach by using the Au binding peptide Z2 (RMRMKMK) [20] and the mild reducing and buffering agent HEPES. Five additional Z2 variants were obtained to study the influence of different modifications to the peptide. The modifications chosen included substitution of methionine residues for isoleucine, the addition of lipid tails with two different lengths, and the addition of a lipid tail accompanied by a reversal of the sequence. A different Au binding peptide, AG3 (AYSSGAPPMPPF), was also included for comparison. Each of the peptides was used to synthesize a set of 64 samples with varying concentrations of peptide, $\mathrm{HAuCl}_{4}$, and HEPES. Each sample was then characterized using UV-Vis spectroscopy. The spectra serve as a fast proxy method for characterizing the nanoparticle structure. That is, although a given spectrum is not a unique representation of a particular nanoparticle structure, we can identify how spectra and therefore structures change as a function of reagent concentrations and peptide identity. We propose an application of functional data analysis methods to the spectral characterizations of plasmonic structures to analyze the high-throughput experimentation data obtained. Finally, we corroborate these differences in nanoparticle synthesis outcomes for each Z2 variant by using a combination of electron microscopy and small-angle scattering, and discuss the significance of changes to peptide chemistry in the context of a large experimental design space. 
Table 1: Peptides used for Au nanoparticle synthesis

\begin{tabular}{l|l|c|c} 
Name & Sequence & Lipid Tail & Reference \\
\hline Z2 & RMRMKMK & & {$[20]$} \\
MZ2 & RMRMKMK & Myristoyl & \\
MZ2R & KMKRMRM & Myristoyl & \\
PZ2 & RMRMKMK & Palmitoyl & \\
Z2M6I & RMRMKIK & & \\
Z2M246I & RIRIKIK & & \\
AG3 & AYSSGAPPMPPF & & {$[21]$}
\end{tabular}

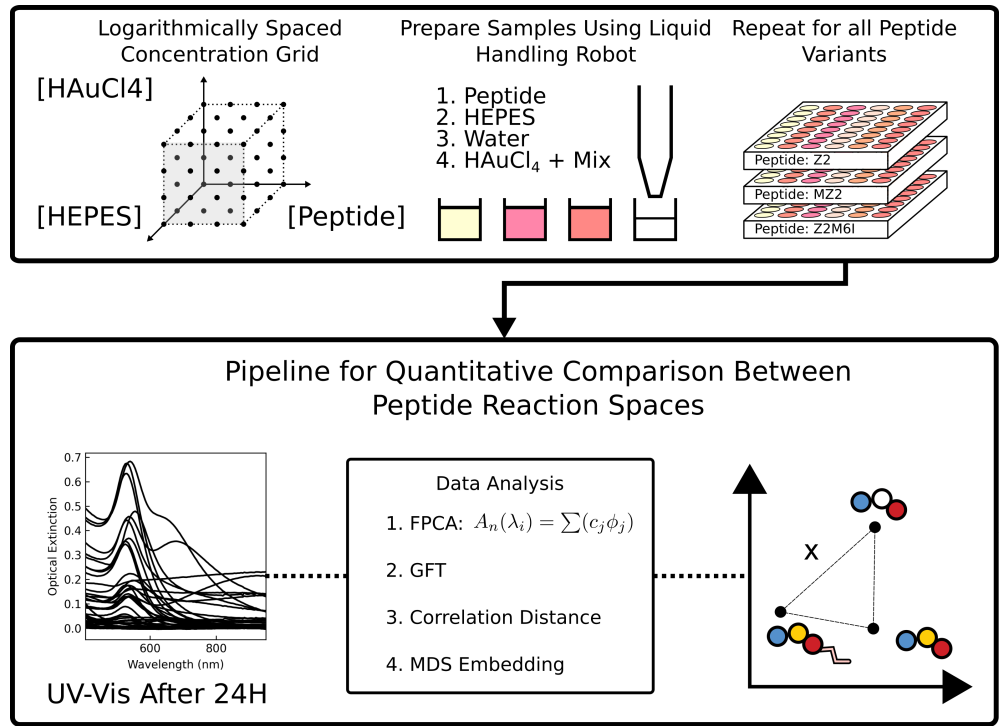

Figure 1: A logarithmically spaced concentration grid of reagents is sampled using a liquid-handling robot. UV-Vis spectra are obtained for each sample and the process is repeated for all 7 peptides. Spectra are then analyzed using the data analysis pipeline to recover a quantitative metric for comparing each peptide's reaction space.

\section{Experimental synthesis and characterization}

\subsection{Materials}

Gold (III) chloride trihydrate (HAuCl4) ( $\geq$ 99.9\%) was obtained from Sigma-Aldrich (St. Louis, MO, USA), 4-(2hydroxyethyl)-1-piperazineethanesulfonic acid (HEPES) was obtained from EMD Chemicals (Gibbstown, NJ, USA), and all peptides were acquired from Biomatik Corporation (Cambridge, ON, Canada). Peptides were purified using HPLC to $>95 \%$ purity by the manufacturer and the lyophilized trifluoroacetate salt was used for experiments without further purification.

\subsection{Sample Preparation}

Stocks of HEPES, HAuCl4, and peptide were prepared by diluting the reagents with ultra pure water obtained from a Direct-Q 3 UV water purification system with a resistivity of 18.2 M $\Omega$ (Millipore Corporation, Bedford, MA, USA). The concentrations of peptide, HEPES, and $\mathrm{HAuCl}_{4}$ stock used were: $5 \times 10^{-2} w t . \%(\mathrm{ca} .0 .5 \mathrm{mM}), 1 \times 10^{-1}$ wt. $\%$ or $1 \times 10^{-2} w t . \%$ (ca. 4.18 or $0.418 \mathrm{mM}$ ), and $1 \times 10^{-1} w t . \%$ (ca. $2.93 \mathrm{mM}$ ), respectively. Synthesis of gold nanoparticles was carried out by pipetting the stocks of peptide, HEPES, additional water, and finally gold chloride into 48-well Falcon microplates (Corning, Corning, NY, USA) using an Opentrons (Brooklyn, NY, USA) OT-2 liquid handling robot. Each solution was pipetted up and down, the microplates were covered, and the samples were left to react at room temperature. 


\subsection{UV-Vis Sample Characterization}

Samples were measured 24 hours after synthesis using a Epoch 2 microplate spectrophotometer (BioTek, Winooski, VT, USA). Background correction was performed by subtracting the absorbance of water. Details of functional data analysis are available in the supporting information.

\subsection{Electron Microscopy}

For imaging on the Scanning Electron Microscope (SEM), $5 \mu \mathrm{L}$ aliquots of samples were deposited on a silicon wafer and air dried overnight. Images were acquired on a FEI (Hilsboro, OR, USA) Sirion XL30 SEM which was typically operated at $5 \mathrm{kV}$ and with a spot size of 3 using through-lens detection and ultra-high resolution mode. Transmission electron microscopy (TEM) samples were prepared by diluting the sample ten-fold and then casting $7 \mu \mathrm{L}$ on a lacey carbon coated film, 200 mesh, copper grids purchased from Electron Microscopy Sciences. Grids were dried in air and imaged on a FEI Tecnai F20 at $200 \mathrm{kV}$.

\subsection{USAXS Measurements and Data Analysis}

Samples were loaded in a custom-made 48-well plate assembly between two Kapton windows for a total sample thickness of $10 \mathrm{~mm}$. USAXS data were collected on the 9-ID-C beamline at the Advanced Photon Source at Argonne National Laboratory using $21 \mathrm{keV}$ X-rays [22]. The USAXS data collection time was 90 seconds, beam size was 0.8 $\mathrm{mm}$ by $0.8 \mathrm{~mm}$, and the flux density was approximately $5 \times 10^{12}$ photons $/ \mathrm{second} / \mathrm{mm}^{2}$. Data were reduced using the standard data reduction protocols and software provided by the beamline (Indira).

Mathematical desmearing based on the Lake method [23] was performed using the provided Igor macros [24] to remove instrumental slit smearing effects in preparation for data fitting. The flat background at high-q was then averaged and subtracted from the data before performing automated Guinier analysis. The latter is an estimate of the radius of gyration $R_{g}$ using the Guinier approximation $I(q)=I(0) \exp \left(\frac{q^{2} R_{g}^{2}}{-3}\right)$. Automated Guinier analysis was performed using AUTORG [25].

\section{Results and Discussion}

\subsection{Effects of Peptide on Au Nanoparticle Synthesis}

Au nanoparticles were synthesized in the presence of one of the 7 peptides listed in Table 1 at 64 different reaction conditions represented by the grid of points in Figure 1. The concentrations of peptide, $\mathrm{HAuCl}_{4}$, and HEPES were sampled at logarithmically spaced intervals. Sample conditions with no peptide or HEPES were also included in this space. We present a portion of the UV-Vis spectra in Figure 2 in which each row from top to bottom corresponds to a decrease in the $\mathrm{HAuCl}_{4}$ concentration level, each column from left to right represents an increasing level of peptide concentration, and the HEPES concentration is fixed at $1 \mathrm{mM}$ (see supporting information for other HEPES levels). These spectra can be qualitatively analyzed as a proxy for nanoparticle structure as a function of reagent conditions and peptide identity. Additionally, optical extinction at $400 \mathrm{~nm}$ can be used to estimate the concentration of $\mathrm{Au}(0)$. However, this method needs to be used with care because extinction at $400 \mathrm{~nm}$ can also be affected by changes in particle shape, size distribution, and the particle's surface chemistry (e.g. adsorption of HEPES or peptide) [26]. The leftmost column in Figure 2 corresponds to the zero peptide condition and is color coded to denote the peptide that was used in the same batch of reactions. HEPES is a mild reducing and buffering agent which forms nanoplates, spherical nanoparticles, or nanostars in the presence of $\mathrm{HAuCl}_{4}$ [27, 28, 29]. HEPES stabilizes nanoparticles against aggregation through its adsorption to the particle surface [30, 31]. As the $\mathrm{HAuCl}_{4}$ concentration increases from $40 \mu M$ to $200 \mu M$ in Figure 2 $(\mathrm{M}, \mathrm{A})$, the ratio of HEPES:HAuCl $\mathrm{H}_{4}$ decreases. This results in the appearance of a second peak and broad extinction at higher wavelengths. This is consistent with formation of nanoplates and particle aggregates as seen in Figure 7D.

The addition of peptide causes significant changes in the spectra for all $\mathrm{HAuCl}_{4}$ levels relative to the no peptide condition. However, the exact changes in spectra vary depending on the peptide identity and concentration. In order to discuss the potential effects of peptides on Au nanoparticle synthesis outcomes, we conceptualized the different ways that peptides can interact with Au precursors and nanoparticles as peptide presentation. Peptide presentation includes, but is not limited to, peptide sequence order, amino acid identity, lipid tail conjugation, charge of residues, solution conditions $(\mathrm{pH}$, ionic strength and identity), proximity of peptide to other peptides, and peptide self-assembly. Peptide presentation in the context of peptide mediated Au nanoparticle synthesis has often been studied by modifying the peptide chemistry - i.e. sequence order, identity, chemical modification, or conjugation of lipid tails to the $\mathrm{N}$-terminus [8, 13, 12]. However, we believe that additional insights and molecular design rules for peptide mediated inorganic nanoparticle synthesis 


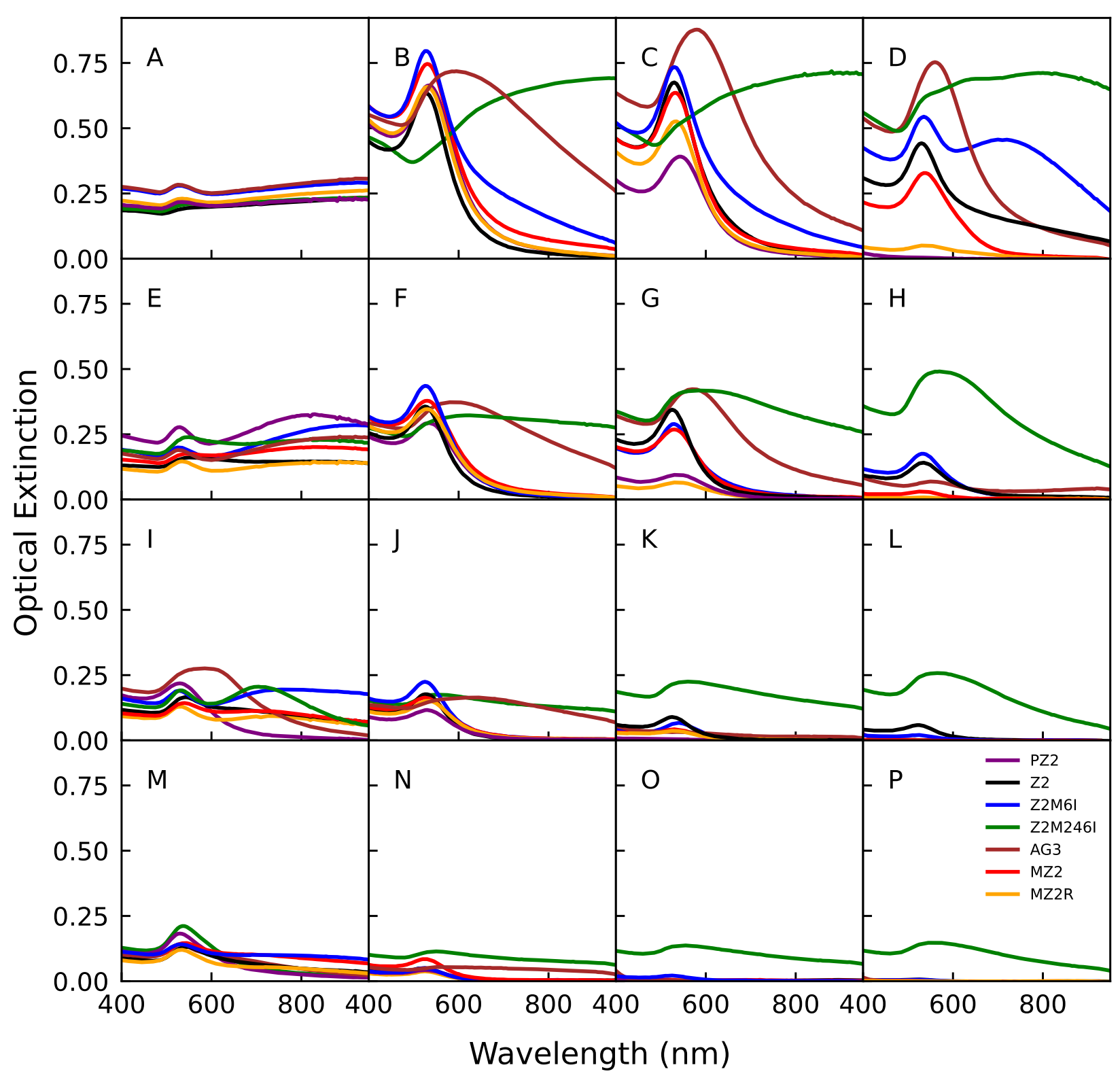

Figure 2: UV-Vis spectra of Au Nanoparticles prepared in the presence of different peptides as indicated by the color labeling. HEPES concentration is $1 \mathrm{mM}, \mathrm{HAuCl}_{4}$ concentration decreases from top to bottom $(0.2,0.117,0.0684,0.04$ $\mathrm{mM})$, and peptide concentration increases from left to right $(0,0.04,0.0894,0.2 \mathrm{mM})$.

may be identified by sampling the effects of modifications to peptides in a large set of reaction conditions with the aid of high-throughput experimentation and new data analysis approaches. We identified Z2 (RMRMKMK) as a good candidate peptide for developing methods to study how peptide presentation affects nanoparticle synthesis. We selected $\mathrm{Z} 2$ because it has a small number of unique amino acids and lacks secondary structure. Unlike many Au binding peptides in the literature which are identified through biopanning methods, Z2 was rationally designed by combining residues with high binding affinities to Au surfaces [20]. Later molecular dynamics simulations concluded that the methionine residues in $\mathrm{Z} 2$ act as anchor residues, which the authors define as having a strong and persistent affinity for the simulated $\mathrm{Au}(111)$ surface [32]. Additionally, methionine is known to complex with $\mathrm{AuCl}_{4}-1$ which would decrease the reduction rate of $\mathrm{Au}$ (III) [9, 33]. We also chose to include AG3 (AYSSGAPPMPPF) in our study in order investigate whether our approach can yield new insights into the similarities and differences of Z2 and AG3 in the context of Au nanoparticle synthesis and stabilization beyond those that have been discussed [11]. 
We observed that at a fixed concentration of $\mathrm{HAuCl}_{4}$ and HEPES, high concentrations of $\mathrm{Z} 2$ and lipidated $\mathrm{Z} 2$ variants led to a decrease in optical extinction at the LSPR peak and at $400 \mathrm{~nm}$. Both of these observations suggest that the concentration of $\mathrm{Au}(0)$ decreases as the concentration of $\mathrm{Z} 2$ and lipidated $\mathrm{Z} 2$ variants is increased [26]. We hypothesized that the complexation of methionine with $\mathrm{HAuCl}_{4}$ is responsible for the decrease in formation of Au nanoparticles after 24 hours. Therefore, we synthesized Z2M6I (RMRMKIK) and Z2M246I (RIRIKIK) to study the effect of partially or completely substituting methionine for isoleucine on peptide-mediated growth of Au nanoparticles. The rationale for choosing isoleucine is that it has similar physicochemical properties to methionine [34], but is not expected to bind to Au surfaces [20, 9] nor complex with $\mathrm{HAuCl}_{4}$. With the exception of Z2M246I, a low concentration of any Z2 variant generally led to more prominent LSPR peaks and lower optical extinction at higher wavelengths when compared to the no peptide condition at the same concentration of HEPES and $\mathrm{HAuCl}_{4}$. An increase in prominence of the the LSPR peak $(\mathrm{ca} .540 \mathrm{~nm})$ is associated with increased formation of spherical nanoparticles and a decrease in extinction at higher wavelengths is consistent with a decrease in aggregation and formation of nanoplates. All samples synthesized in the presence of Z2M246I on the other hand, had significant and broad optical extinction at wavelengths higher than the typical peak position of an LSPR peak associated with spherical Au nanoparticles. Additionally, samples prepared with Z2M246I were visually distinct from samples prepared with the other Z2 variants in this study. The former sedimented and had a green or black hue, which indicated the formation of large particles or aggregates. Whereas the latter were typically pink, purple, or red and remained dispersed. We also observed that increasing the concentration of Z2M6I or Z2M246I did not lead to a significant decrease in optical extinction at $400 \mathrm{~nm}$. Therefore we concluded that the structures formed in the presence of Z2M246I should be most distinct from others in the study, and that complexation of $\mathrm{HAuCl}_{4}$ with methionine was an important factor in controlling the proportion of $\mathrm{Au}(\mathrm{III})$ that was reduced to $\mathrm{Au}(0)$ after 24 hours.

Several previous works have investigated the use of a lipid tail conjugated to AG3 to control the in-situ formation of Au nanoparticle decorated peptide nanostructures (e.g. micelles, fibrils) [12, 13, 14, 15, 16, 17]. The addition of an alkyl tail to a water soluble peptide makes it amphiphilic, and therefore capable of self-assembling in water. Once self-assembled, peptide nanostructures may act as a template for nucleation or adsorption of Au nanoparticles. Most uses of amphiphilic peptides in the synthesis of Au nanoparticles use just one or a limited set of reaction conditions to draw conclusions about the effect of peptide modifications to Au nanoparticle synthesis outcomes. We were interested in gaining a deeper understanding of the role of peptide lipidation on Au nanoparticle synthesis by using amphiphilic peptides in a large range of reaction conditions. Increasing the length of lipidated peptide tails is one method for decreasing the critical micelle concentration. However, peptide self-assembly can also be affected by the ratio of precursor to peptide due to interactions between peptide residues and the precursor. Ionic interactions between $\mathrm{AuCl}_{4}{ }^{-}$ and positively charged residues and complexation may lead to changes in the critical micelle concentration and the structure of the peptide-precursor assembly. Therefore, in addition to making substitutions to the Z2 sequence, we also obtained three variants which were modified at the N-terminus with either a palmitic acid tail (PZ2), myristic acid tail (MZ2), or a myristic acid tail with a reversed sequence (MZ2R). SAXS studies of MZ2 dissolved at a concentration of $4.2 \mathrm{mM}$ revealed extended structures which could be fit with a cylindrical model, and are therefore likely to be cylindrical micelles. However, this concentration is much larger than $0.2 \mathrm{mM}$, the highest concentration used in $\mathrm{Au}$ nanoparticle synthesis experiments. We used pyrene fluorescence to estimate the critical micelle concentration of MZ2, MZ2R, and PZ2 to be $0.47 \mathrm{mM}, 0.33 \mathrm{mM}$, and $0.24 \mathrm{mM}$, respectively (see supporting information) [35]. Note that the conditions used in this work are all below the critical micelle concentration. Therefore, the study is limited to the interaction of dissolved peptides with particle surfaces, and does not include the growth of Au over self-assembled peptides. In the case of Z2, reversing the sequence maintains the position of the methionines but swaps the positions of lysines and arginines with one another. The physicochemical similarity of arginine and lysine may suggest that swapping their positions in a sequence should lead to negligible changes in the peptide's affinity to Au surfaces and Au nanoparticle synthesis outcomes. However, unlike lysine, arginine has been classified as an anchor residue in sequences other than Z2, and the first arginine in the Z2 sequence was close to being classified as a contact residue by Tang et al. [32]. Therefore, we were interested in whether changing the position of lysine and arginine relative to the lipid tail would lead to an appreciable change in Au nanoparticle synthesis outcomes.

The spectra of samples prepared in the presence of lipidated peptides were generally similar to that of the unmodified Z2. However, unlike Z2, the lipidated variants led to a more significant decrease in overall optical extinction as the peptide concentration increased. Additionally, unlike the non-lipidated peptides in this study, an increase in optical extinction at higher wavelengths for high peptide concentrations was not observed for the lipidated Z2 variants. Had the lipidated peptides led to structural organization of nanoparticles, a red-shift and peak broadening should be observed due to plasmonic coupling [1]. Therefore, we interpreted the lack of optical extinction at higher wavelengths as an indication that the lipidated Z2 variants were unlikely to be organizing Au nanoparticles. However, we expected that the presence of $\mathrm{HAuCl}_{4}$ should reduce the concentration of lipidated peptide required for its self assembly. As will be discussed in a later section, peptide-precursor structures do exist in the sample, but these do not lead to organized peptide nanostructures decorated by nanoparticles. The decrease in optical extinction for lipidated peptides relative 
to non-lipidated peptides is likely due to a decrease in the Au reduction rate. A decrease in Au reduction rate could be caused by the peptide-precursor interactions forming structures that inhibit access of HEPES to Au species. This explanation is supported by the fact that samples prepared with PZ2 had lower optical extinction than those prepared with MZ2. The longer length of the palmitic acid tail should lead to stronger association between peptides, and therefore more strongly inhibit reactions of HEPES with Au species. The spectra of Au nanoparticles formed in the presence of MZ2R were comparable to those formed with either MZ2 or PZ2, with the distinction that MZ2R inhibited Au nanoparticle formation to a greater extent than MZ2, and less than PZ2. This observation is in line with the order of critical micelle concentrations estimated for the three peptides. Overall, changing the positions of arginine and lysine in the sequence did not lead to a significant change in the peptide's function in modulating Au nanoparticle synthesis. Therefore, the weak impact of this modification should be reflected in later analyses which use the full synthesis space to make pairwise comparisons between peptides. The spectra from samples synthesized in the presence of AG3 show clear LSPR peaks in most conditions, but these are red-shifted and broadened relative to those synthesized in the presence of Z2. These spectral changes are consistent with formation of larger particles and aggregation. Colloidal instability of AG3 capped particles has been correlated with acidic conditions, the ratio of reducing agent to precursor, and the ratio of peptide to precursor [8]. In contrast to the conditions used in this work, other publications which use HEPES, the AG3 sequence, and modified AG3 variants, utilize higher HEPES concentrations such as 0.1 M HEPES [15]. The differences between synthesis conditions in which Z2 and AG3 form colloidally stable nanoparticles reveal high dissimilarity between the two sequences.

\subsection{Comparison of Peptide Mediated Nanostructure Formation Using the Full Parameter Space}

Each peptide's synthesis space, consisting of the $4 \times 4 \times 4$ set of reagent concentrations and their respective spectra, is a rich dataset of nanoparticle formation outcomes. We posit that leveraging the full dataset would strengthen our analysis of each peptide's effect on nanoparticle formation. To accomplish this, we use each peptide's synthesis space in an unsupervised learning approach to study correlations between each peptide. Comparing the synthesis space of peptides would then require comparing the set of spectra along with their sample concentrations. One crucial aspect of this comparison is the way one represents the synthesis space for data analysis purposes. To correlate different synthesis spaces of peptide sequences, we first parameterize the sampled synthesis space by a graph (i.e. a network of first-order connections) using the different component concentrations as nodes and edges obtained using a $k$-nearest neighbors approach. The above parameterization results in a natural data representation for the synthesis space via signals on the graphs where the signal corresponds to the UV-Vis characterization for the size and shape of Au nanoparticles among others. For example, for a synthesis space comprising of concentration of peptide, buffer, and $\mathrm{HAuCl}_{4}$, each sample (a node in the graph) in the synthesis space can be considered as a point in a three-dimensional space. Euclidean distance between the sample concentrations can then be used to obtain edges in the graph using a $k$-nearest neighbors construction. For each sample in the synthesis space, its characteristic UV-Vis response is collected between the wavelength region of 400-900 $\mathrm{nm}$ with $5 \mathrm{~nm}$ increments. Given that response of each sample (i.e. the signal at each node of the graph) is a function $x(\lambda)$ (mapping a given wavelength to an absorption intensity) rather than an array of values typically considered in data analysis, we use a principal component analysis (PCA) adapted for functional data to obtain vector-like descriptors for each sample [36, 37]. The approach referred to as FPCA and the graph based signal processing used in this paper are described in the supporting information. In the next few subsections, we describe the application of these methods to UV-Vis spectroscopy data collected from the high-throughput sampling described earlier.

\subsection{Application of FPCA to UV-Vis Spectroscopy}

In this section, we demonstrate application of functional data analysis (FDA) to spectral data collected from UV-Vis spectroscopy (see Section 7.1 for more details). Following the notation in the supporting information, the UV-Vis spectroscopy data is denoted as $x(\lambda)$ where $\lambda$ is a wavelength. Given a set of UV-Vis spectroscopy data, we obtain a matrix $x_{i j}$ (where $i$ is a sample and $j$ is a wavelength), and compute its principal components highlighting types of variations present. For seven peptides with 64 samples in each synthesis space, we have a total of $N=448$ samples. We apply FPCA to all the UV-Vis samples collected for our study and observe that $\approx 95 \%$ of the functional variance is captured using only 8-components. A qualitative understanding of each FPCA component can be obtained by generating spectra while varying the component using Equation (1) as the linear generative model. In Figure 3, we plot spectra generated from each one of four components where we sample one standard deviation away from the mean (dotted black curve) in positive (blue curve) and negative direction (red curve). We observe that the obtained FPCA components are informative in the following sense: i) moving one standard deviation away from the mean along PC 1 direction changes the peak from being non-existent to a sharp peak thus signifying the primary signal regarding formation of gold nanoparticles. ii) Similarly, PC 2 can be associated with both a peak shift and a change in broad optical extinction at higher wavelengths, which could indicate changes in particle aggregation. iii) PC 3 corresponds to a shift along the 
intensity axis which we associate with changes in $\mathrm{HAuCl}_{4}$ concentration under the conditions that forms nanoparticles. iv) We also observe that rest of components are redundant in terms of the information they encode at the spectra level. For example, PC 4 can be considered as mirroring the features encoded in PC 2 while PC 5 is a scaled copy of PC 3. Further more, PC 6 can be associated with a combination of PC 2 and PC 3; PC 7 and 8 provide minimal changes to the spectra when generated from the model. The above qualitative method is used to further decrease the components to just four given by the top four eigenvalues. For the rest of this paper, we represent each UV-Vis spectra $x(\lambda)$ using its first four principal component scores $f_{i}, i=[1,2,3,4]$ resulting in a vector representation of a signal on graph parameterization of grid-like synthesis space (see Section 7.2 in supporting information for more details on this parameterization).

We compare synthesis space of peptides using a notion of similarity between the signals obtained over the synthesis space. For UV-Vis spectra collected over the synthesis space, the signals can now be replaced by their corresponding FPCA representation resulting in a vector descriptor for each node in the graph parameterization. Vector signals on graphs are easier to process mathematically using the linear algebra techniques extensively developed for graphs [38, 39]. However, it is not straightforward as to how to define a similarity function between two graph signals. Defining a graph $G=(V, E)$ as set of vertices $V$, edges $E$ associated with a signal $S: V \rightarrow \mathbb{R}^{4}$, we need a way to compare two signals $S_{i}, S_{j}$. One approach to achieve this is to compress the graph signal into a vector by a change of basis using a graph Fourier transform (GFT) described in Section 7.2. GFT allows us to a) compress the graph signal into a vector; $b$ ) remove noise in the signal; making it a robust choice of data representation.

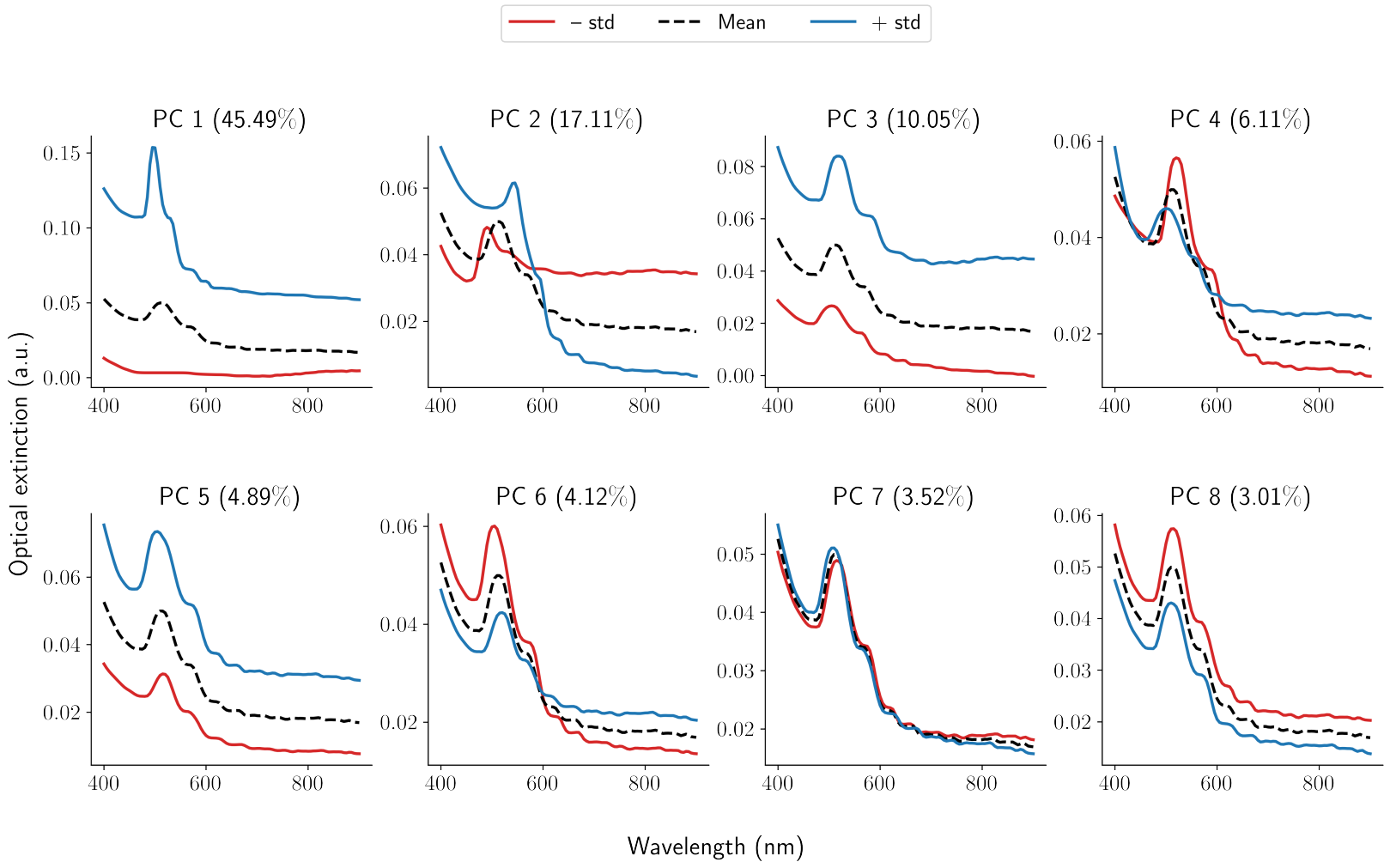

Figure 3: Generated samples from Equation (1). Each panel corresponds to a principal component annotated on the top along with corresponding explained variance. For each component, the mean of the functions is denoted using a black-dotted curve while positive and negative standard deviations along the component are denoted using blue and red curves respectively.

\subsection{Application of GFT to High-Throughput Experimentation Data}

In this section, we describe an application of GFT to represent the data collected for each peptide. Suppose $G=(V, E)$ is a graph constructed from Euclidean embedding of component concentrations. We represent each sample's UV-Vis spectra using a vector of FPCA score values and obtain a graph signal $S: V \rightarrow \mathbb{R}^{4}$ at each node. We then use GFT on $S$ and retain the lowest $70 \%$ of frequencies resulting in $\hat{S}$. Given a pair of peptides $i, j$, we compare their synthesis spaces 
by computing distance between $\hat{S}_{i}, \hat{S}_{j}$. A similarity measure such as the correlation distance (Equation 3 ) is used to quantify similarities between synthesis space i.e. types of Au nanoparticles formed when we vary the concentrations in synthesis space. Figure 4 is the result of application of GFT represented signals $\hat{S}$ for multi-variate analysis of peptide data. On the left panel of Figure 4, we used a correlation distance (see Equation (3) between compressed graph signal
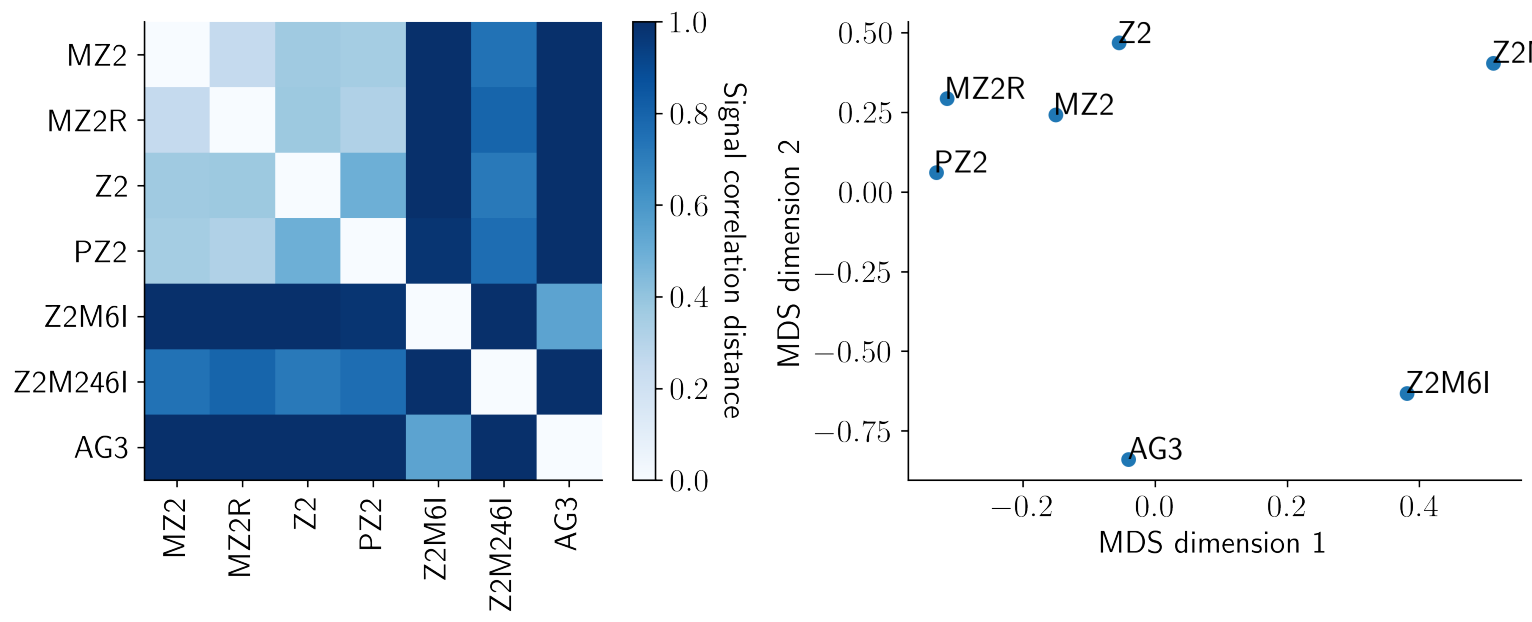

Figure 4: Application of graph Fourier transform to high-throughput experimentation data with an FPCA representation of UV-Vis spectra. (left) a correlation distance between graph signal representation in Fourier domain i.e. $\hat{S}$ of different peptides; (right) a metric embedding of the similarity measure shown on the left

of pairs of peptides and represented the similarities using a heatmap plot. The similarity between synthesis spaces is depicted using a continuous color gradient light blue (highly similar) and dark blue (highly dissimilar). A metric embedding using multi-dimensional scaling [40, Chapter 14.8] of the correlation distance is shown on right. We observe that changes to our base peptide Z2 result in two distinct modes of synthesis space. Namely, changes to the type of alkyl chain (MZ2, PZ2) and reversing the sequencing (MZ2R) result in very similar synthesis space while changes to actual peptide sequence (AG3, Z2M6I, Z2M246I) result in a diverse synthesis space as quantified by distances in the metric embedding shown in the right panel of Figure 4 where the two groups are clearly separated.

\subsection{Structural Characterization Using Electron Microscopy and USAXS}

The correlation distances describe how each pair of peptides differs in the effect it has on Au nanoparticle synthesis across the whole synthesis space. However, it is important to validate the conclusions we drew from UV-Vis spectra by more direct structural characterization methods.

We repeated the synthesis of Au nanoparticles in the presence of each of the $\mathrm{Z} 2$ variants in a slightly modified synthesis space $\left(6 \times 4 \times 2\right.$, peptide $\mathrm{x} \mathrm{HAuCl}_{4} \mathrm{x}$ HEPES). The bounds of this synthesis space were the same as those used for UV-Vis characterization, but the number of peptide concentrations studied was increased to more finely assess the impact of peptides on synthesis. The HEPES concentrations was 0 or $1 \mathrm{mM}$ to study peptide-precursor structure on its own and because this concentration of HEPES led to more colloidally stable samples than those formed at lower concentrations, respectively. The USAXS scattering profiles presented in Figure 5 show the results for all peptide Z2 variants. Even in the absence of HEPES, most of the scattering profiles show a Guinier region. This is consistent with the formation of hypothesized co-assembled peptide-precursor structures.

The scattering profiles of all $\mathrm{Z2}$ variants in the presence of $\mathrm{HAuCl}_{4}$ and no HEPES are presented in Figure $5 \mathrm{~A}$ and B. The scattering profiles of Z2M246I were similar to that of other Z2 variants which had methionine. Therefore, we concluded that the studied peptide variants co-assemble with the precursor due to ionic interactions between the positively charged residues (arginine and lysine) and $\mathrm{AuCl}_{4}{ }^{-1}$, and not due to methionine- $\mathrm{HAuCl}_{4}$ complexation.

In the presence of $1 \mathrm{mM}$ HEPES the scattering profiles of lipidated peptides more closely resembled one another than any of the non-lipidated variants. This can be seen in Figure $5 \mathrm{C}$ and D, in which samples which were lipidated show a Guinier region and do not have signs of colloidal instability, unlike samples prepared with non-lipidated peptides. The scattering profiles of non-lipidated peptides in Figure $5 \mathrm{D}$ indicate aggregation or the presence of large particles due to the upturn in scattering at low-q. The Guinier region of samples prepared with lipidated peptides in $1 \mathrm{mM}$ HEPES was fit using AUTORG [25]. The results are presented using a $\log -\log$ plot of $R_{g}$ as a function of the peptide: $\mathrm{HAuCl}_{4}$ 


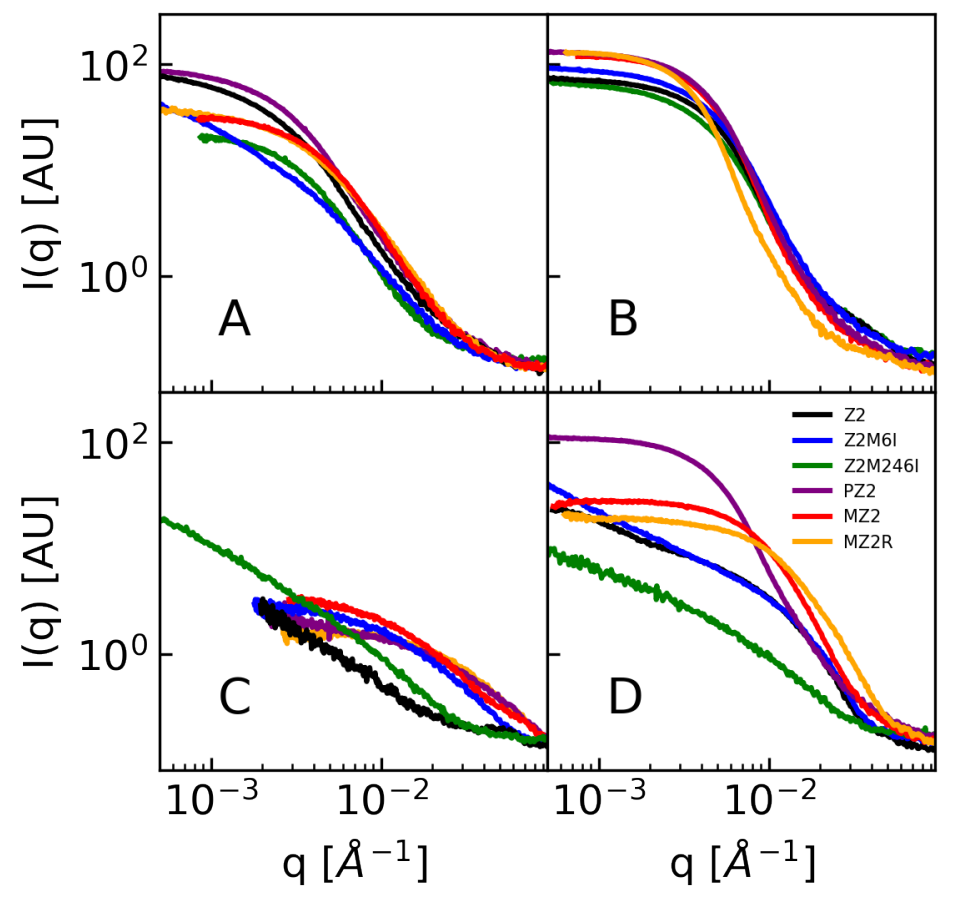

Figure 5: A subset of smeared USAXS data. Remaining data is available in the supporting information. Concentrations of peptide, $\mathrm{HAuCl}_{4}$, and HEPES: A 0.04, 0.2, $0 \mathrm{mM}, \mathrm{B} 0.2,0.2,0 \mathrm{mM}, \mathrm{C} 0.04,0.2,1 \mathrm{mM}, \mathrm{D} 0.2,0.2,1 \mathrm{mM}$. Note that while most samples show a Guinier region in the absence of HEPES, non-lipidated peptides in the presence of HEPES and $\mathrm{HAuCl}_{4}$ do not have a Guinier region and may be colloidally unstable.

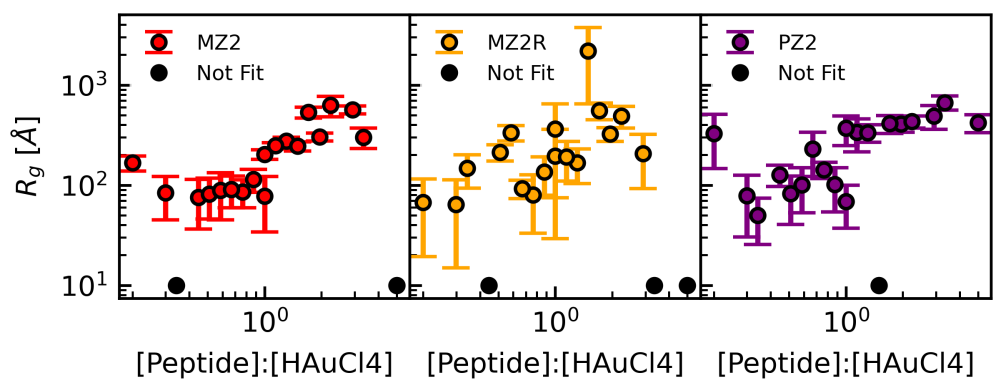

Figure 6: Radius of gyration results obtained from AUTORG plotted as a function of peptide: $\mathrm{HAuCl}_{4}$ molar ratio for all lipidated peptides and a HEPES concentration of $1 \mathrm{mM}$. Error bars indicate standard deviation. Profiles which did not contain a Guinier region that could be fitted were marked using black and plotted at a fixed $R_{g}$ of $10 \AA$. $R_{g}$ fit results for all peptides in the absence of HEPES are available in the supporting information. Data of non-lipidated peptides in the presence of $1 \mathrm{mM}$ HEPES is not presented because the majority of samples did not have a Guinier region. 

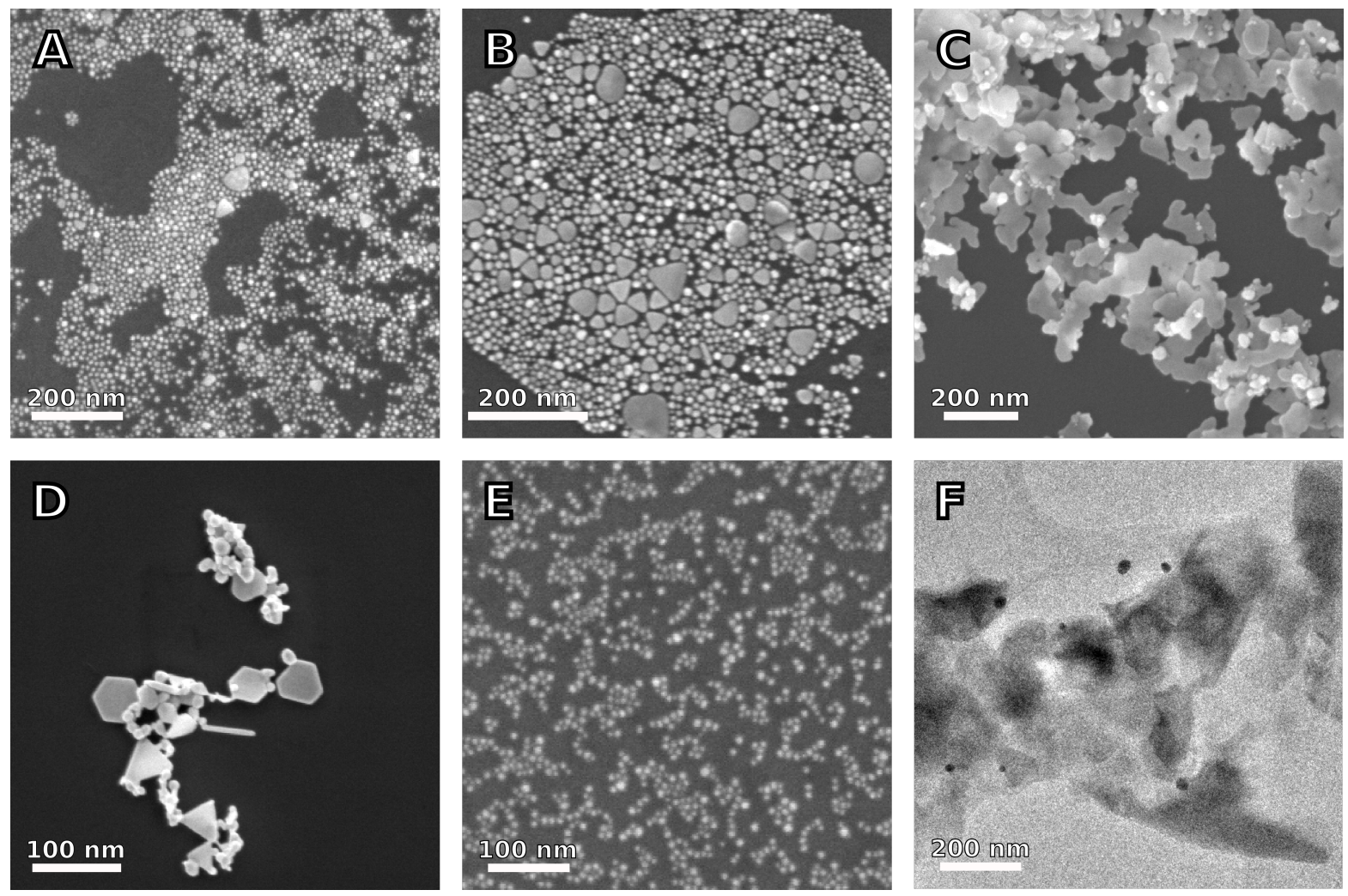

Figure 7: Electron microscopy results. A, B, and C: SEM micrographs of Au nanoparticles prepared in the presence of Z2, Z2M6I, and Z2M246I, respectively. The reagent concentrations for these samples were $0.04 \mathrm{mM}$ peptide, $1 \mathrm{mM}$ HEPES, and $0.2 \mathrm{mM} \mathrm{HAuCl}_{4}$. D: aggregated Au nanoparticles and nanoplates synthesized in $1 \mathrm{mM} H E P E S$ and 0.2 $\mathrm{mM} \mathrm{HAuCl}_{4}$. E and F: SEM and TEM micrographs of samples prepared in $1 \mathrm{mM} \mathrm{HEPES,} 0.2 \mathrm{mM} \mathrm{HAuCl}_{4}$ and 0.04 or $0.2 \mathrm{mM} \mathrm{MZ2R}$, respectively.

molar ratio in Figure 6. Samples which could not be fit with a Guinier region are shown as black markers with a $R_{g}$ of $10 \AA$. All three lipidated $\mathrm{Z} 2$ variants show an increase in $R_{g}$ as a function of peptide: $\mathrm{HAuCl}_{4}$ ratio. The majority of samples prepared with non-lipidated peptides and $1 \mathrm{mM}$ HEPES did not contain a Guinier region due to aggregation or sedimentation of larger particles and could therefore not be analyzed using AUTORG. The similarity of the scattering profiles of samples prepared with lipidated peptides is in line with the analysis of the signal correlation distance of peptide synthesis spaces. Drawing conclusions from how non-lipidated peptides affected the formation of $\mathrm{Au}$ nanoparticles across the synthesis space is precluded by colloidal instability. Nevertheless, future high-throughput experimentation studies of nanoparticle synthesis may utilize small-angle scattering for systems which lack an easy to measure proxy of structure (i.e. LSPR). Fitting of scattering profiles provides a low-dimensional descriptor of the structure (the fit parameters) which can be used to optimize a particular structure as a function of experimental conditions [41]. Alternatively, these model fitting results could be used to compare how sequence-defined molecules or other additives impact nanoparticle synthesis. However, small-angle scattering data fitting is an ill-posed problem and challenges with automated data analysis are continuing to be studied. Challenges that preclude us from applying more complex and automated data fitting to our dataset are the presence of mixed particle morphologies and sample sedimentation during longer than normal measurement times when queuing large numbers of samples.

Next, we used SEM and TEM to study the structure of a small subset of samples formed in the presence of different Z2 variants. The SEM image in Figure 7]A shows that the sample synthesized in the presence of Z2 contains both spherical nanoparticles and nanoplates. However, the sample contains fewer nanoplates and is less aggregated than the sample in Figure 7 D, which corresponds to the same reagent concentration but with no peptide. Samples prepared with lipidated peptides such as MZ2R contained no plate like particles as seen in Figure 7]E and F. Additionally, the particles formed in the presence of MZ2R appear to be more monodisperse relative to the particles formed in the presence of $\mathrm{Z} 2$. The spectra which correspond to the reagent concentrations with and without peptides in these micrographs are shown in Figure 2A and B, respectively. These SEM images support our previous interpretation of the spectra that the addition of $\mathrm{Z} 2$ leads to less aggregation and fewer nanoplates. Although the presence of nanoplates in the sample prepared with 
Z2 is not apparent from Figure 2 B, higher concentrations of Z2 and Z2M6I did show an increase in optical extinction at higher wavelengths and are likely to contain more plate like structures.

Samples prepared with the lipidated peptide MZ2R in Figure 7 E, on the other hand, contain no plate like particles. However, as was previously discussed, lipidation of Z2 was not sufficient to lead to formation of nanoparticle decorated peptide nanostructures. This is due to the fact the peptide concentrations in this study are below the critical micelle concentration. Additionally, Z2 has several charged amino acid groups and is therefore more likely to assemble into less extended or even spherical micelles [42]. Future work into using Z2 to control the assembly of Au nanoparticles could focus on the use of a lipid tail that is longer than palmitic acid, or the addition of hydrophobic amino acids to the sequence [12]. Using a higher peptide concentration of $0.2 \mathrm{mM}$ caused sample charging in SEM. Therefore, TEM was used to characterize samples formed in the presence of MZ2R at this higher concentration. The image in Figure $7 \mathrm{~F}$ shows what appears to be a large amorphous aggregate with Au nanoparticles adsorbed on its surface. We interpret this as further evidence of peptide-precursor aggregation. However, the size of these amorphous aggregates is larger than what is to be expected from the fits in Figure 6, and the structures are therefore likely to be affected by drying.

The sample prepared in the same conditions as Figure 7 A, but with the Z2M6I and Z2M246I peptides instead of Z2, are shown in Figure 7 B and C, respectively. The sample prepared with Z2M6I is similar to that prepared with Z2, with the exception that it contains a higher fraction of plate-like particles. This increase in plate-like particles is consistent with Z2M6I having higher extinction than Z2 at wavelengths above the main LSPR peak, as can be seen in Figure 2 B. The SEM image of the sample prepared with Z2M246I primarily contains flat, and interconnected structures. These interconnected structures also have smaller particles deposited on top, but the shape of these smaller particles is not clear. The large structures are similar to other reported flat and branching structures whose formation mechanism was attributed to molecular confinement [43]. However, the reason for Z2M246I leading to similar structures remains unclear and remains to be further studied. The differences in particle morphology observed in samples containing Z2M6I and Z2M246I support the high signal correlation distances between these peptides and the other Z2 variants presented in Figure 4

We summarize our main findings in Figure 8 , where we show that each of the $\mathrm{Z} 2$ peptide variants formed peptideprecursor aggregates in the absence of HEPES. Given that $\mathrm{HAuCl}_{4}$ is added last to the solution, we infer that peptideprecursor aggregation is a process that happens in conjunction with Au reduction. High concentrations of peptide variants which contained methionine showed a significant decrease in the amount of $\mathrm{Au}(0)$ formation after 24 hours based on comparison of optical extinction at $400 \mathrm{~nm}$ relative to lower peptide concentrations. We note that this change cannot be explained by an increase in the amount of peptide adsorbed to the surface given that adsorption of organic molecules to citrate capped nanoparticles resulted in an increase in optical extinction [26]. In the case of the lipidated peptides in this study, the difference in to what extent $\mathrm{Au}(0)$ formation was suppressed was inversely proportional to the values of critical micelle concentrations. Therefore, the differences in $\mathrm{Au}(0)$ formation in the presence of lipidated peptides are likely due to differences in modulation of $\mathrm{HAuCl}_{4}$-methionine complexation and availability of $\mathrm{HAuCl}_{4}$ to HEPES. These differences arise due to variation in the strength of lipidated peptide-precursor interactions between MZ2, MZ2R, and PZ2. Z2M6I and Z2M246I have one or all three methionine residues substituted with isoleucine, respectively. In Z2M6I, the relative proportion of plate-like particles to spherical particles appears to increase relative to Z2. Whereas for Z2M246I the structures that formed are interconnected and branching plate-like structures. Finally, the optical extinction at $400 \mathrm{~nm}$ for Z2M246I is least sensitive to peptide concentration out of all of the peptides studied and therefore confirms that methionine is an important modulator of $\mathrm{HAuCl}_{4}$ reduction.

\section{Conclusion}

In this paper, we presented a data analysis pipeline for studying large synthesis spaces using high-throughput experimentation. We characterize the formation of Au nanoparticles in the presence of peptides using our high-throughput experimentation framework to sample large synthesis spaces and collect UV-Vis spectra for each sample as a proxy signal for the nanoparticle structure. We then used a series of data representation and compression techniques to discern the correlation between peptides based on their full synthesis space. Using metrics designed to quantify higher-order correlations and variations between features, we obtain a two-dimensional embedding that provides insights into the similarity between the effect that peptides have on Au nanoparticle synthesis. The framework presented here is generic and only assumes the synthesis space being sampled is continuous. We applied the proposed framework to six variants of the Au binding peptide Z2 and a different Au binding peptide AG3. We obtained a Euclidean embedding of the correlations revealing that the synthesis spaces are more affected by the peptide sequence than conjugation of a lipid tail. The conclusions of this analysis were supported by use of USAXS and electron microscopy, which are lower-throughput but more direct methods of structural characterization. We anticipate that the proposed approach will initiate further research aiming at analyzing high-throughput experimentation data with spectral characterization to explore and understand complex synthesis spaces. There are two notable limitations of the current method: i) 


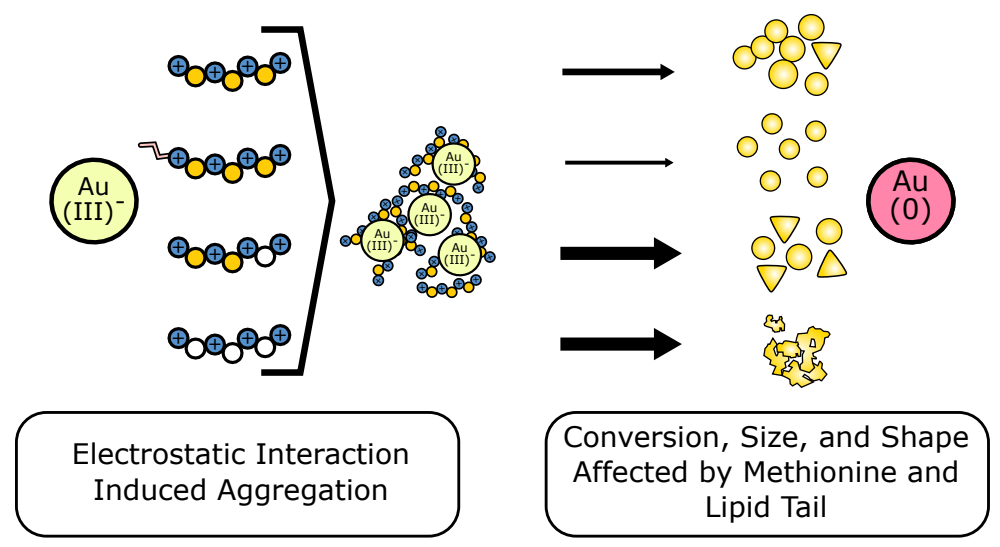

Figure 8: Schematic showing the effects of Z2 and its variants on Au nanoparticle synthesis outcomes. All Z2 variants were shown to form peptide-precursor aggregates based on the USAXS data in the absence of HEPES. We inferred that this aggregation occurs due to attractive electrostatic interactions between $\mathrm{AuCl}_{4}{ }^{-1}$ and the positively charged residues present on all Z2 variants, shown above as blue circles. Next, we use thinner and thicker arrow to indicate lower and higher conversion of $\mathrm{Au}(\mathrm{III})$ to $\mathrm{Au}(0)$ in the $\mathrm{Z} 2$ variants, respectively. Conversion was least affected by presence of peptide variants which had methionine (yellow circles) substituted for isoleucine (white circles) (Z2M6I, Z2M246I). Conversion was significantly decreased in the presence of lipidated peptide variants (MZ2, MZ2R, PZ2). Finally, we show how these modifications of Z2 led to differences in nanoparticle size and shape. In short, Z2 lipidation led to more dispersed spherical nanoparticles, and substitution of methionine increased anisotropic particle growth relative to the base $\mathrm{Z} 2$ sequence.

Although the principal components capture interpretable variations in Au nanoparticle spectra, the analysis method cannot attribute these variations to physically meaningful variables (i.e. reagent concentrations or physicochemical peptide attributes). This can, for example, be improved by using the PCA features in causal models [44, 45]. ii) The PCA data representation limits the variations of spectra to linear cases such as peak intensities. Alternatively, data representations that characterize spectra using disentangled low dimensional representations would provide a direct way of visualizing variations in the synthesis space and their similarities across.

\section{Acknowledgements}

This material is based upon work supported by the US Department of Energy (DOE), Office of Science, Office of Basic Energy Sciences (BES), as part of the Energy Frontier Research Centers program: CSSAS - The Center for the Science of Synthesis Across Scales - under Award Number DE-SC0019288. Part of this work was conducted at the Molecular Analysis Facility, a National Nanotechnology Coordinated Infrastructure (NNCI) site at the University of Washington, which is supported in part by funds from the National Science Foundation (awards NNCI-2025489, NNCI-1542101), the Molecular Engineering \& Sciences Institute, and the Clean Energy Institute. This research used resources of the Advanced Photon Source, a U.S. Department of Energy (DOE) Office of Science User Facility, operated for the DOE Office of Science by Argonne National Laboratory under Contract No. DE-AC02-06CH11357. Extraordinary facility operations were supported in part by the DOE Office of Science through the National Virtual Biotechnology Laboratory, a consortium of DOE national laboratories focused on the response to COVID-19, with funding provided by the Coronavirus CARES Act. We would like to thank Jan Ilavsky and Ivan Kuzmenko for assistance in remote experiments at APS. Part of this work was conducted with instrumentation provided by the Joint Center for Deployment and Research in Earth Abundant Materials (JCDREAM).

\section{Supplementary}

\subsection{Materials}

Pyrene (98.2 \% purity) was obtained from Sigma-Aldrich and used without further purification. 


\subsection{Determination of Lipidated Peptide Critical Micelle Concentration}

The pyrene stock was prepared by first preparing a $0.5 \mathrm{mM}$ stock solution of pyrene in ethanol. The ethanol solution was diluted with deionized water to reach a pyrene concentration of $0.146 \mathrm{mg} / \mathrm{mL}$. Note that the final ethanol concentration was 0.144 vol.\%. Peptide stock solutions were prepared at $5 \times 10^{-1} w t . \%$ and $5 \times 10^{-2} w t$. $\%$. Samples were prepared using an Opentrons OT-2 liquid handling robot. Solutions were prepared by transferring peptide, pyrene stock, and additional water into Caplugs Evergreen untreated 96-well microplates (black, flat bottom). The concentration of peptide in the samples was varied from $0.04 \mathrm{mM}$ to $3 \mathrm{mM}$. Samples were mixed by pipetting up and down after addition of water, and were then characterized using a BioTek Synergy H1 microplate reader. Samples were excited at $334 \mathrm{~nm}$ and emission was obtained at 372 (peak 1) and $384 \mathrm{~nm}$ (peak 3).

Pyrene remains dissolved in samples where the peptide concentration is too low for formation of micelles. Above the critical micelle concentration (CMC), pyrene adsorbs to the peptide lipid tails inside micelles. The resulting change in the polarity of pyrene's environment leads to changes in its fluorescence emission. The ratio between emission peaks 3 and 1 as a function of peptide concentration is used to estimate the critical micelle formation [35, 46, 47]. The CMC is estimated from the intersection of two lines in the plot of the ratio of the two fluorescence peaks as a function of the logarithm of peptide concentration. The order of the resulting CMC values are MZ2 > MZ2R > PZ2.
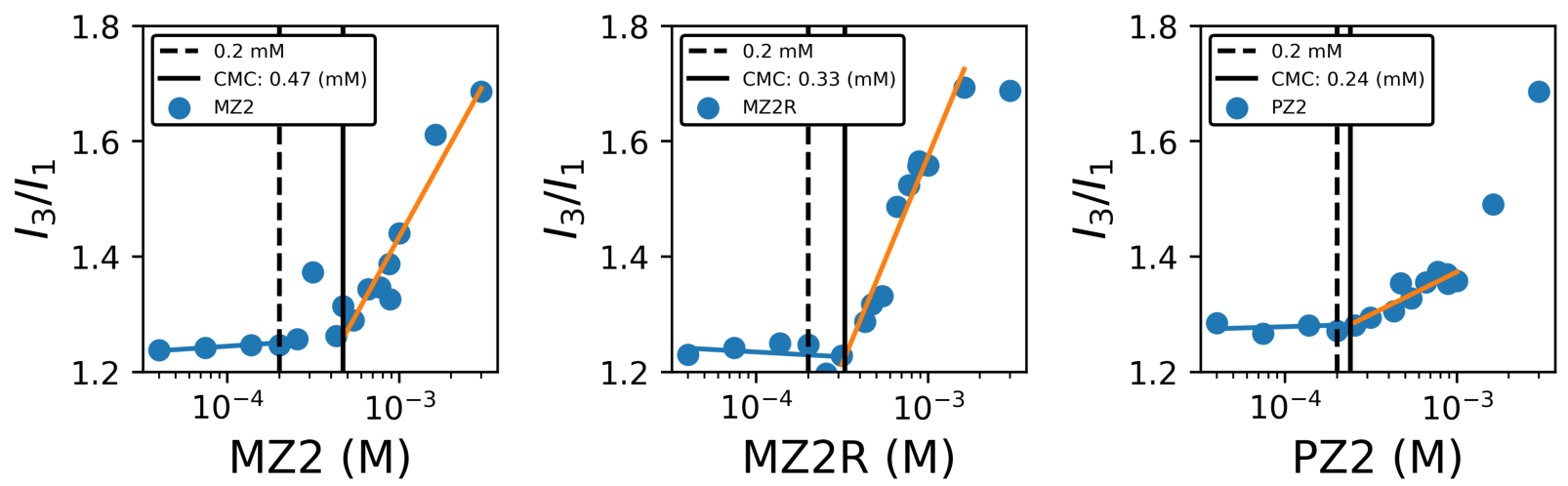

Figure 9: Ratio of fluorescence intensity at 384 to $372 \mathrm{~nm}$ resulting from excitation at $334 \mathrm{~nm}$ as a function of peptide concentration. Adsorption of pyrene to micelles leads to a change in the fluorescence spectra which is used to estimate the CMC by finding intersection of two lines fitted to the data. The CMC of MZ2, MZ2R, and PZ2 were estimated to be $0.47 \mathrm{mM}, 0.33 \mathrm{mM}$, and $0.24 \mathrm{mM}$, respectively.

\section{Functional Data analysis}

In this section, we describe the mathematical details of our framework.

\subsection{Functional Principal Component Analysis}

Similar to standard PCA used in the multivariate analysis of high-dimensional vectors, functional principal component analysis (FPCA) aims to represent each data point (a function) in terms of its variation from the mean [36, 37]. One advantage of using PCA-based representation of the data is that dimensionality can be reduced to represent types or modes of variation. This is especially useful for UV-Vis spectra where the expected variations are low in number such as peak position, amplitude, etc. The goal in PCA is to obtain dominant (or principal) variations of the data (from the mean) by solving for a set of weight vectors that maximize variance along different components called principal components. Let $d$ discrete evaluations of a function $x(t)$ represented as $x_{i j}$ for $j=1,2,3, \ldots, d$ be the $i^{\text {th }}$ data point of the functional data $\mathcal{F}$ for $i=1,2,3, \ldots, N$. Similar to multi-variate PCA for vector-like data, FPCA assumes an underlying linear model for the variations of $\mathcal{F}$ :

$$
f=\mu+\beta x
$$

where $\mu$ is the mean of samples $x_{i}$ and $\beta$ is the learnable weight matrix corresponding to an orthogonal basis for the linear model. The covariance of the data $V(s, t)$ can now be estimated using:

$$
V(s, t)=N^{-1} \sum_{i}^{N} x_{i}(s) x_{i}(t)
$$


The linear model of $\mathcal{F}$ along any given component $f_{i}=\mu+\beta_{i} * x_{i}$ highlights a particular type of variations of sample $x_{i}$ along a component $\beta_{i}$. The principal components $\beta$ highlighting variations of $\mathcal{F}$ are then optimized by maximizing $\langle\beta, V \beta\rangle$ where $\langle\cdot, \cdot\rangle$ is the Euclidean inner-product. Following the terminology of multi-variate PCA, the values $f_{i}$ are called scores for the optimized principal components $\beta(t)$. We illustrate usage of functional PCA using an example case study below where the samples from $\mathcal{F}$ are a set of functions $x(t)=c \sin (2 \pi t)$ for $c \in[0,1]$ and $t \in[0,1]$ as shown in Figure 10. The samples have only one mode of variation (i.e. one dimension of functional variation) in the amplitude highlighted using the blue color gradient in Figure 10.

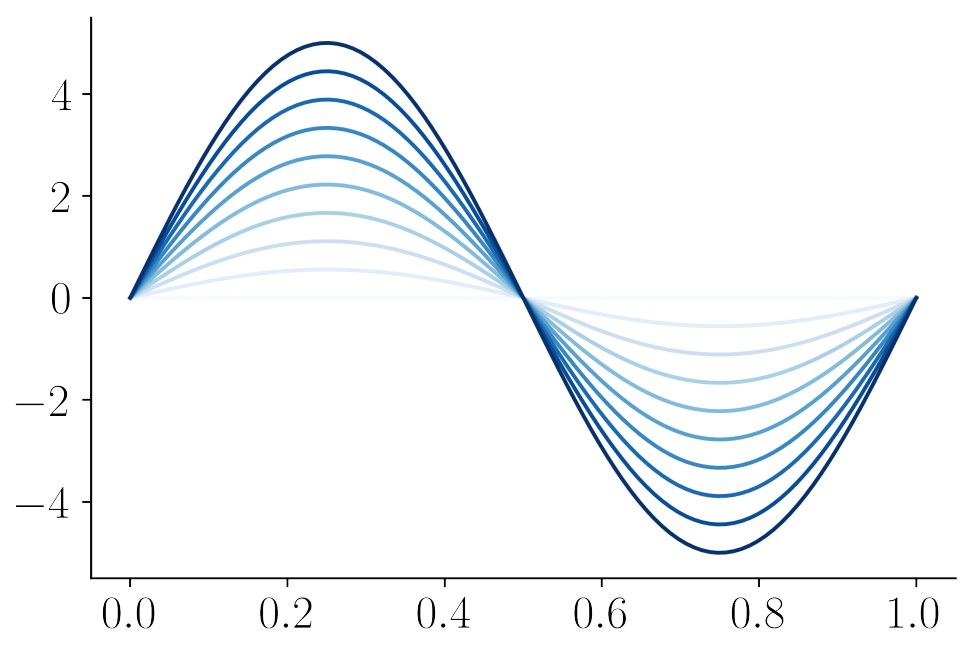

Figure 10: Synthetic data generated with $f(t)=c \sin (2 \pi t)$ with $c \in[0,1]$

Because the FPCA is a linear generative model, we can draw samples from it using principal component scores. Figure 11 depicts the only principal component along with the mean function (in red solid line) and a percentage indicating the total amount of variation accounted for by each component. The grey gradient solid lines in Figure 11 represent linear spaced sample draws from the trained FPCA model for the functional data in Figure 10 We observe that for any given sample draw $f_{i}=\mu+\alpha \beta_{i}$ from the FPCA model, the value $\alpha$ changes the peak amplitude which is the only variation present in $\mathcal{F}$.

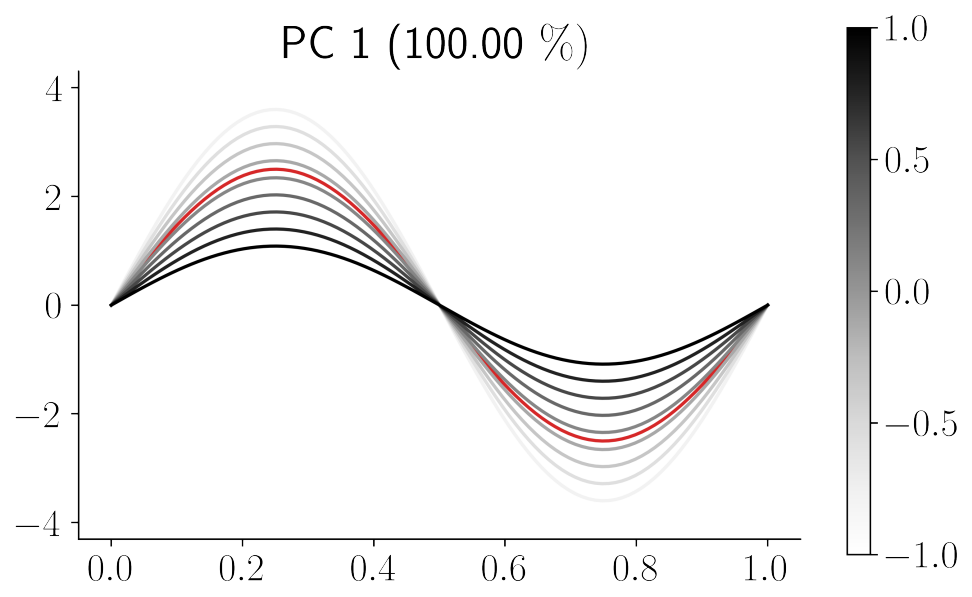

Figure 11: Samples generated from the Principal component 1 of FPCA using Equation 11 with mean of the data in Figure 10 shown in the solid red line.

In this paper, we use a class of FPCA (called joint-FPCA) introduced in [48] that can efficiently decouple the variations along $\mathrm{x}$ and $\mathrm{y}$ axis of one dimensional functional data. We refer interested readers to [48] and [37, Chapter 8.9] as the details are beyond the scope of this paper. 


\subsection{Graph Fourier Transforms (GFT)}

Graph Fourier Transforms (GFT) [38, 39] offer a way to decompose signals indexed by nodes/vertices into a weighted combination of different frequencies on a given graph structure. Given a spatial embedding of a graph, the frequencies correspond to different oscillations of a signal. Mathematically, the GFT defines an irreducible representation of signal space $\mathcal{S}$ with the basis given by the eigenfunctions of the graph Laplacian matrix $L$ representing variations of signals defined on the graph vertices. The graph Laplacian is defined as $L:=D-A$ where $D$ is the degree matrix and $A$ is the adjacency matrix. Given a signal $S: V \rightarrow \mathbb{R}^{d}$, the Laplacian operator $L$ computes the signal variation from local average:

$$
(L s)_{i}=\left(\frac{1}{D_{i i}} \sum_{i j \in E} s_{j}\right)-s_{i}
$$

Using the spectral theorem for symmetric matrices [49], we can obtain a orthogonal basis for the linear operator $L_{n \times n}$ in terms of eigenfunctions $\phi_{0}, \phi_{1}, \ldots, \phi_{n}$ for a graph with $n$ nodes. Motivated by interpretation of Fourier basis as the eigenfunctions of the second derivative operator on a circle, the Laplace eigenfunctions are associated with oscillations on an irregular domain such as a graph. GFT $\hat{S}$ in the eigenbasis of $L$ for signal $S$ can be obtained using:

$$
\hat{S}(k)=\sum_{i \in V} S(i) \phi_{k}
$$

Figure 12 below shows an example using GFT on a circle where different eigenfunctions of a circle (represented as a graph) are shown using a color code.
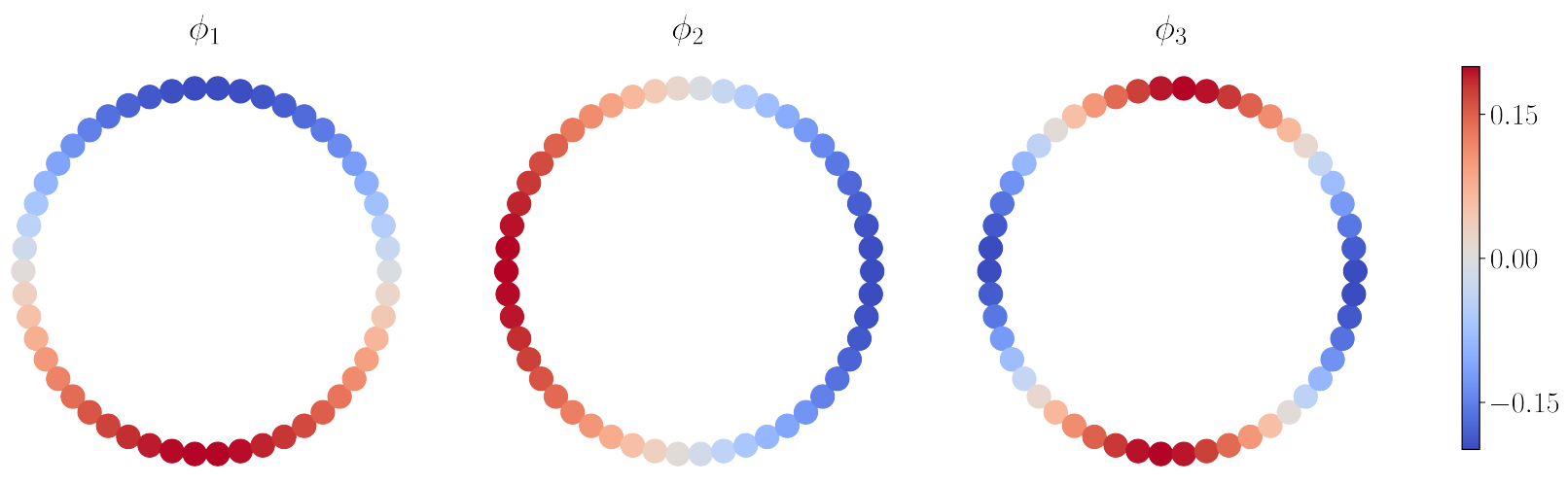

Figure 12: Eigen-functions $\phi_{i}$ for $i=1,2,3$ for a circle

We recover the standard Fourier basis in one-dimensions in Figure 13 by plotting the eigenbasis in Figure $5 b$ with vertex index (i.e. $\theta$ ). Any signal defined over the vertices of a circle can then be represented as a sum of different $\phi_{i}$ in (see Figure 12) multiplied by a scalar using Equation (2) analogous to one-dimensional Fourier representation. Once the graph signal is represented using the Fourier transform, we can then obtain a flattened vector where each dimension correspond to a combination of the original signal dimension and frequency. A distance between two Fourier transformed graph signals $\hat{S}_{i}, \hat{S}_{j}$ can then be computed using:

$$
d=1-\frac{\left(\hat{S}_{i}-\overline{\hat{S}_{i}}\right) \cdot\left(\hat{S}_{j}-\overline{\hat{S}_{j}}\right)}{\left\|\left(\hat{S}_{i}-\overline{\hat{S}}_{i}\right)\right\|_{2}\left\|\left(\hat{S}_{j}-\overline{\hat{S}}_{j}\right)\right\|_{2}}
$$

where ${ }^{-}$and $\|\cdot\|_{2}$ represent the mean and norm of the signal. Two signals are considered identical if the distance $d$ is zero and the similarity decreases with increase in $d$.

\subsection{UV-Vis Spectra}

Note that although Z2M6I contains an outlier in the 0 mM HEPES condition shown in Figure 14]J, the use of GFT on the transformed spectra would have treated this sample as noise. 


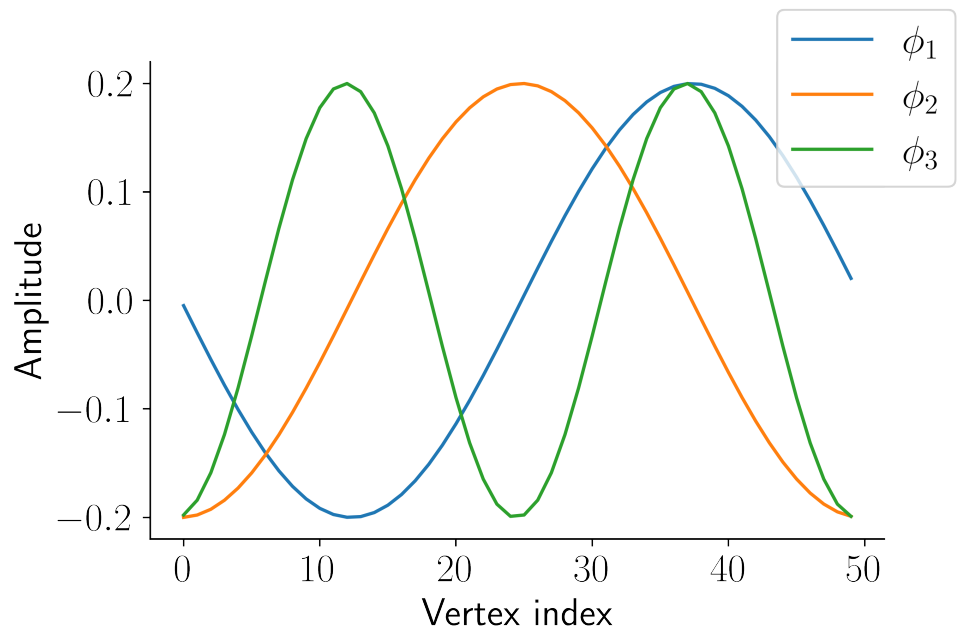

Figure 13: Eigen-functions of the circle graph in Figure 12 visualized as one dimensional functions over vertex index

\subsection{USAXS Scattering Profiles and AUTORG Results}

The AUTORG results for lipidated and non-lipidated peptides are presented in Figure 19 and Figure 20, respectively. The lipidated and non-lipidated peptide- $\mathrm{HAuCl}_{4}$ assemblies are of similar size across all of the studied conditions. However, the size of the lipidated variants appears to vary less as a function of the peptide: $\mathrm{HAuCl}_{4}$ ratio when compared with the non-lipidated variants. Note in Figure 20 that the data for peptides which are lacking methionine showed similar scattering behavior to the $\mathrm{Z} 2$ peptide. We interpret this to mean that peptide- $\mathrm{HAuCl}_{4}$ interactions that are responsible for the formation of the observed aggregates are not due to methionine- $\mathrm{HAuCl}_{4}$ complexation.

\subsection{SAXS}

MZ2 (myristoylated RMRMKMK) was dissolved at a concentration of $4.2 \mathrm{mM}$ in ultra pure water. The sample was flowed into a quartz capillary ( $1 \mathrm{~mm}$ outer diameter, Charles Supper Company, Natick, MA). The capillary was loaded inside a Kratky-type SAXS instrument (SAXSess, Anton Paar, Graz, Austria) with a $\mathrm{Cu} K \alpha$ source $(\lambda=1.54$ $\AA$ ). The slit-smeared scattering data was fit using a slit-smeared cylinder model using SASview (SasView v.5.0.1, www.sasview.org) [50, 51, 52]. The resulting radius of the fitted cylinder model was $5.2 \mathrm{~nm}$. The length could not be determined from the scattering data due to the lack of a Guinier region in the q-range of the instrument. Therefore we can only conclude that the length of the extended, self-assembled MZ2 structures was in excess of $60 \mathrm{~nm}$.

\subsection{USAXS Sample Holder Assembly}

The liquid sample holder used for USAXS experiments is a sandwiched well-plate style assembly which has the same profile as a standard well plate. Files and instructions for assembly have been shared online [53]. The process of assembly is as follows:

1. Insert o-rings into center piece face which will be facing the X-ray source.

2. Place Kapton sheet and then the aluminum cover piece which will be facing the source on top of the o-rings.

3. Insert screws and fasten in the order specified in the image.

4. Turn over the plate and insert o-rings.

5. Load liquid samples manually or with a liquid-handling system. Suggested volume is $450 \mu L$.

6. Place second Kapton sheet and aluminum cover plate on top, and fasten using the same order of screws.

\subsection{Opentrons Protocol Details}




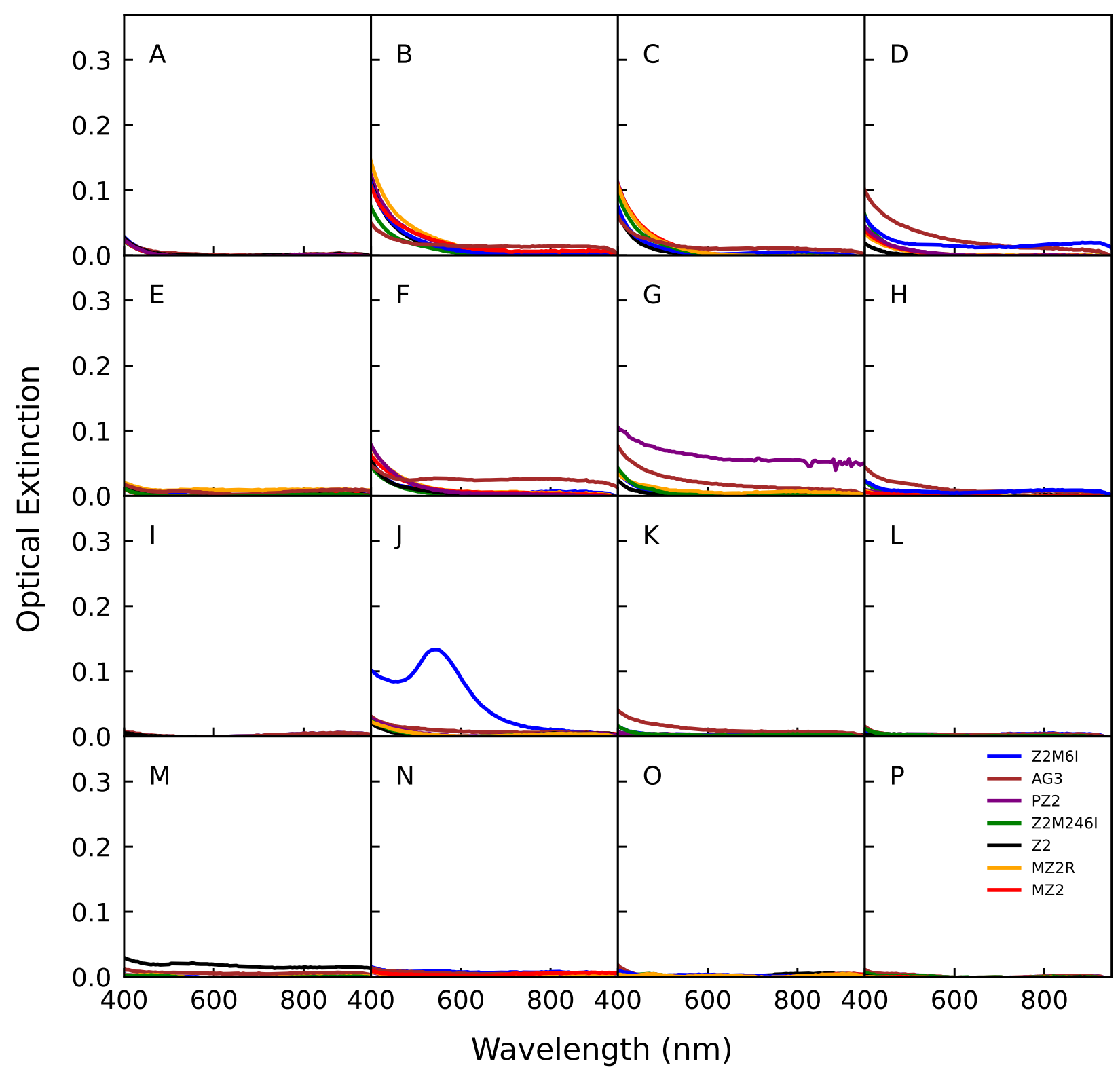

Figure 14: UV-Vis spectra of Au Nanoparticles prepared in the presence of different peptides as indicated by the color labeling. HEPES concentration is $0 \mathrm{mM}, \mathrm{HAuCl}_{4}$ concentration decreases from top to bottom $(0.2,0.117,0.0684,0.04$ $\mathrm{mM})$, and peptide concentration increases from left to right $(0,0.04,0.0894,0.2 \mathrm{mM})$. 


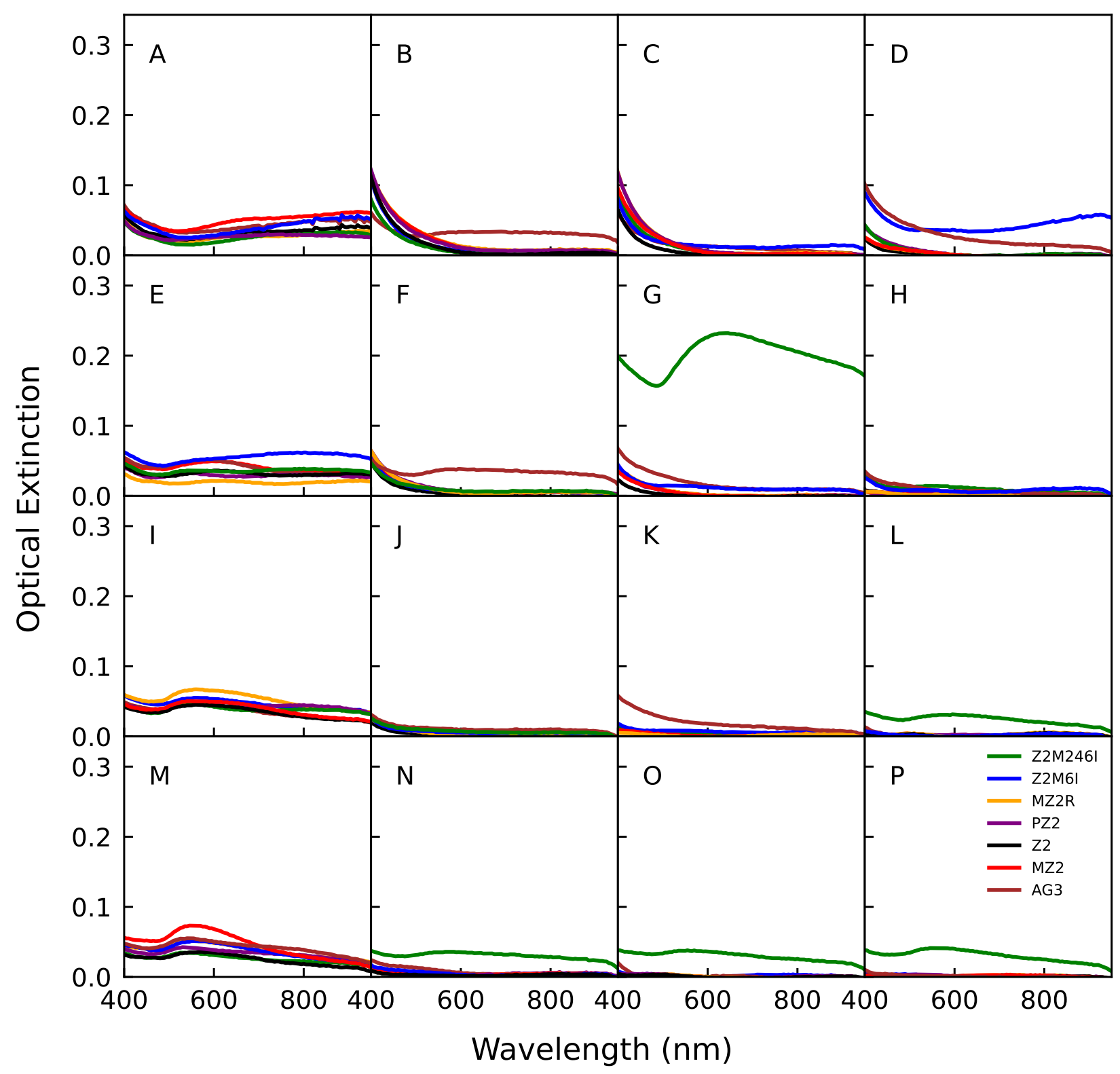

Figure 15: UV-Vis spectra of Au Nanoparticles prepared in the presence of different peptides as indicated by the color labeling. HEPES concentration is $0.01 \mathrm{mM}, \mathrm{HAuCl}_{4}$ concentration decreases from top to bottom $(0.2,0.117,0.0684$, $0.04 \mathrm{mM})$, and peptide concentration increases from left to right $(0,0.04,0.0894,0.2 \mathrm{mM})$. 


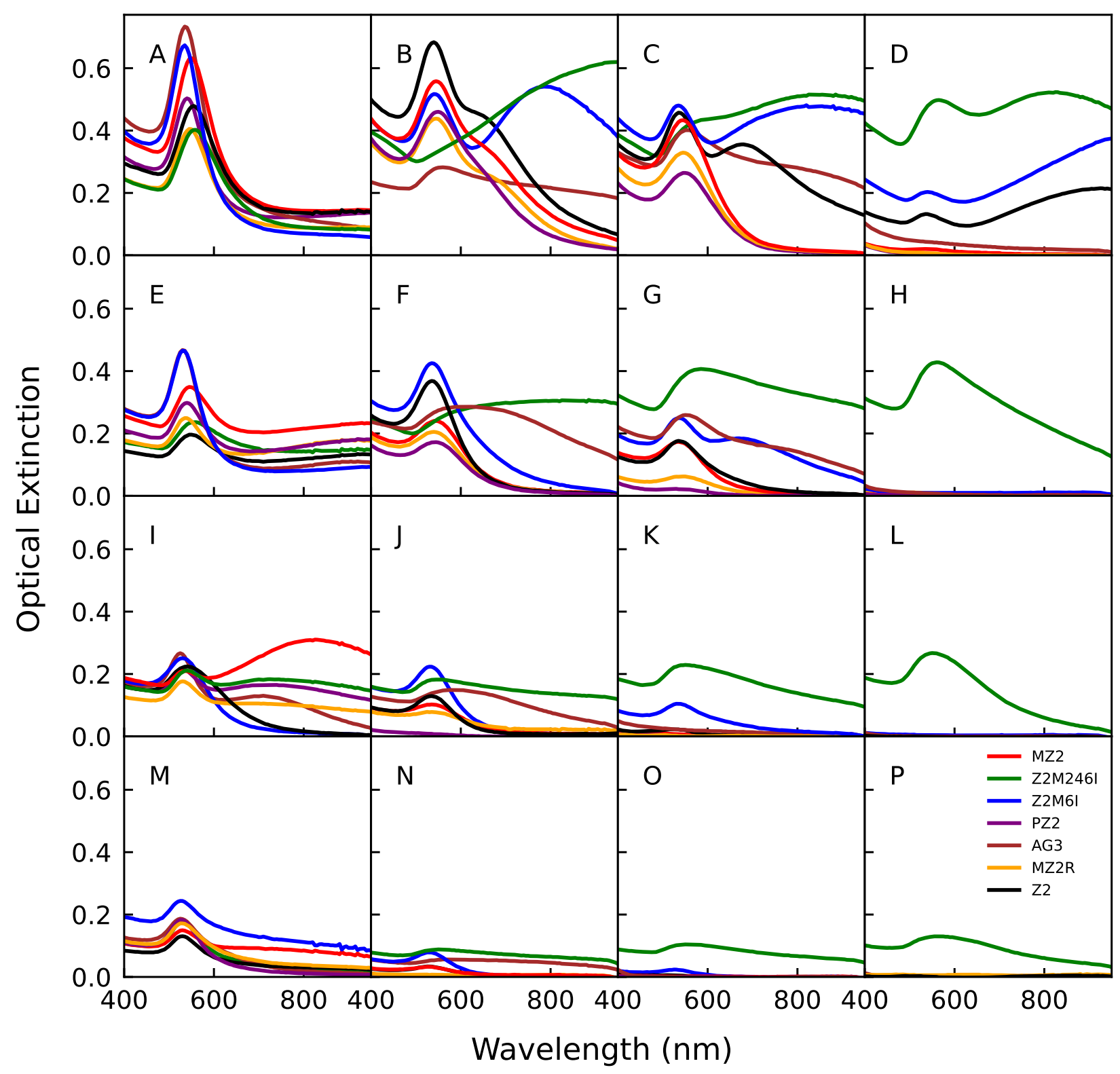

Figure 16: UV-Vis spectra of Au Nanoparticles prepared in the presence of different peptides as indicated by the color labeling. HEPES concentration is $0.1 \mathrm{mM}, \mathrm{HAuCl}_{4}$ concentration decreases from top to bottom $(0.2,0.117,0.0684$, $0.04 \mathrm{mM})$, and peptide concentration increases from left to right $(0,0.04,0.0894,0.2 \mathrm{mM})$. 


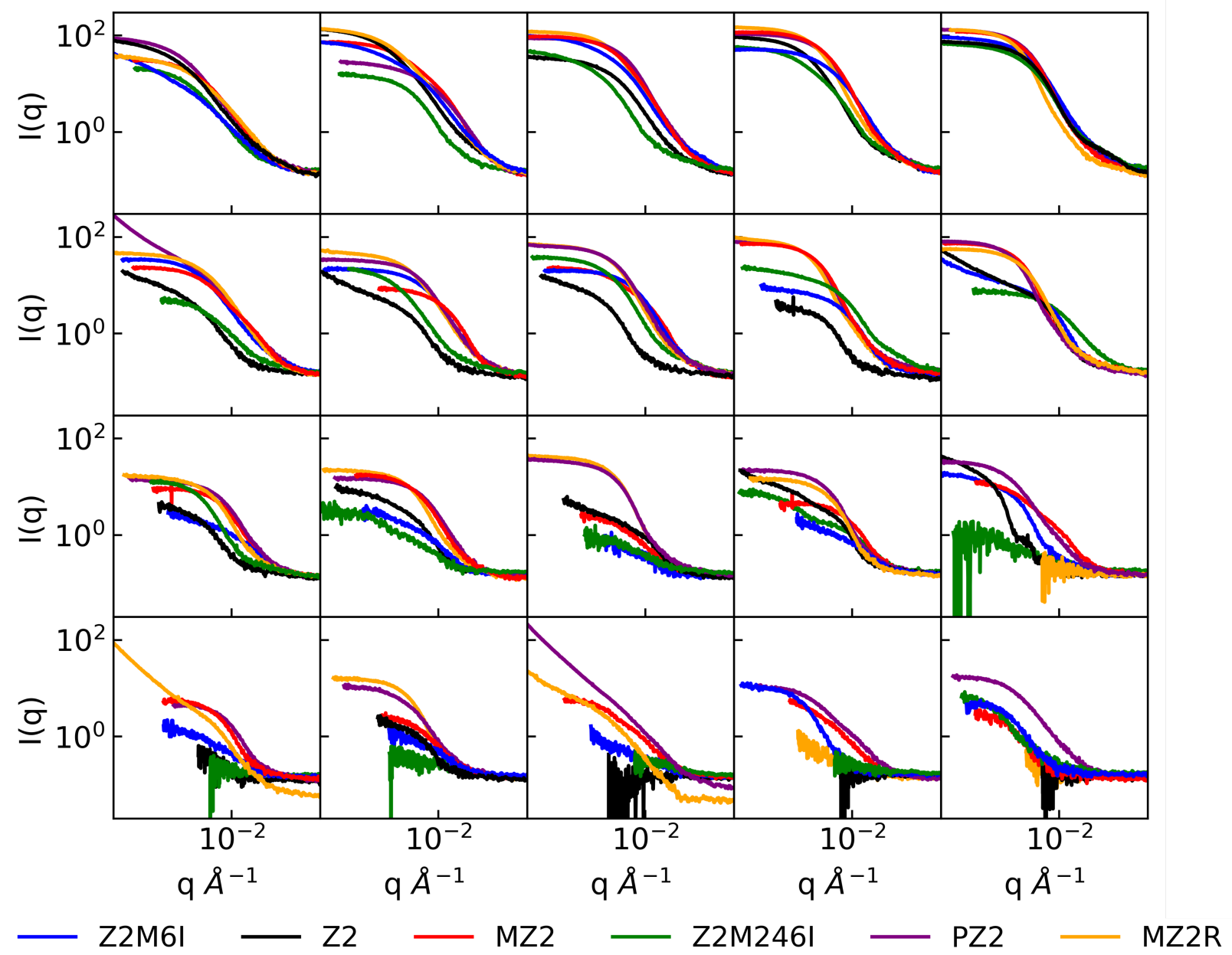

Figure 17: Smeared USAXS data. Sample compositions: 0 mM HEPES; left to right 0.040, 0.060, 0.089, 0.134, and $0.200 \mathrm{mM}$; top to bottom $0.200,0.117,0.068$, and $0.040 \mathrm{mM}$.

Table 2: List of items used per plate

\begin{tabular}{l|l|l|l|c}
\hline Supplier & $\begin{array}{l}\text { Material and Dimen- } \\
\text { sions }\end{array}$ & Catalog Number & Part Name & Qty. \\
\hline Formlabs & Clear or Gray & $\begin{array}{l}\text { RS-F2-GPCL-04 or } \\
\text { RS-F2-GPGR-04 }\end{array}$ & Center piece/main body & 1 \\
\hline McMaster-Carr & Aluminum & $9140 T 109$ & Cover plate source & 1 \\
\hline McMaster-Carr & Aluminum & 9140 T109 & Cover plate detector & 1 \\
\hline McMaster-Carr & Kapton (0.0010" thick) & 2271K401 & Windows & 2 \\
\hline McMaster-Carr & $\begin{array}{l}\text { Buna-N or EPDM (1.5 } \\
\text { mm wide, 9 mm ID) }\end{array}$ & $\begin{array}{l}\text { 9262K126 } \\
1289 N 319\end{array}$ & O-rings & 96 \\
\hline McMaster-Carr & $\begin{array}{l}\text { Stainless steel M3 (8 } \\
\text { mm Long, 0.5 mm } \\
\text { Pitch) }\end{array}$ & 90666 A104 & Screws & 18 \\
\hline MiSUMi & $\begin{array}{l}\text { Brass Cadmium-Free } \\
\text { (M3x0.5 mm) }\end{array}$ & FB-305580 & Threaded insert & 9 \\
\hline
\end{tabular}




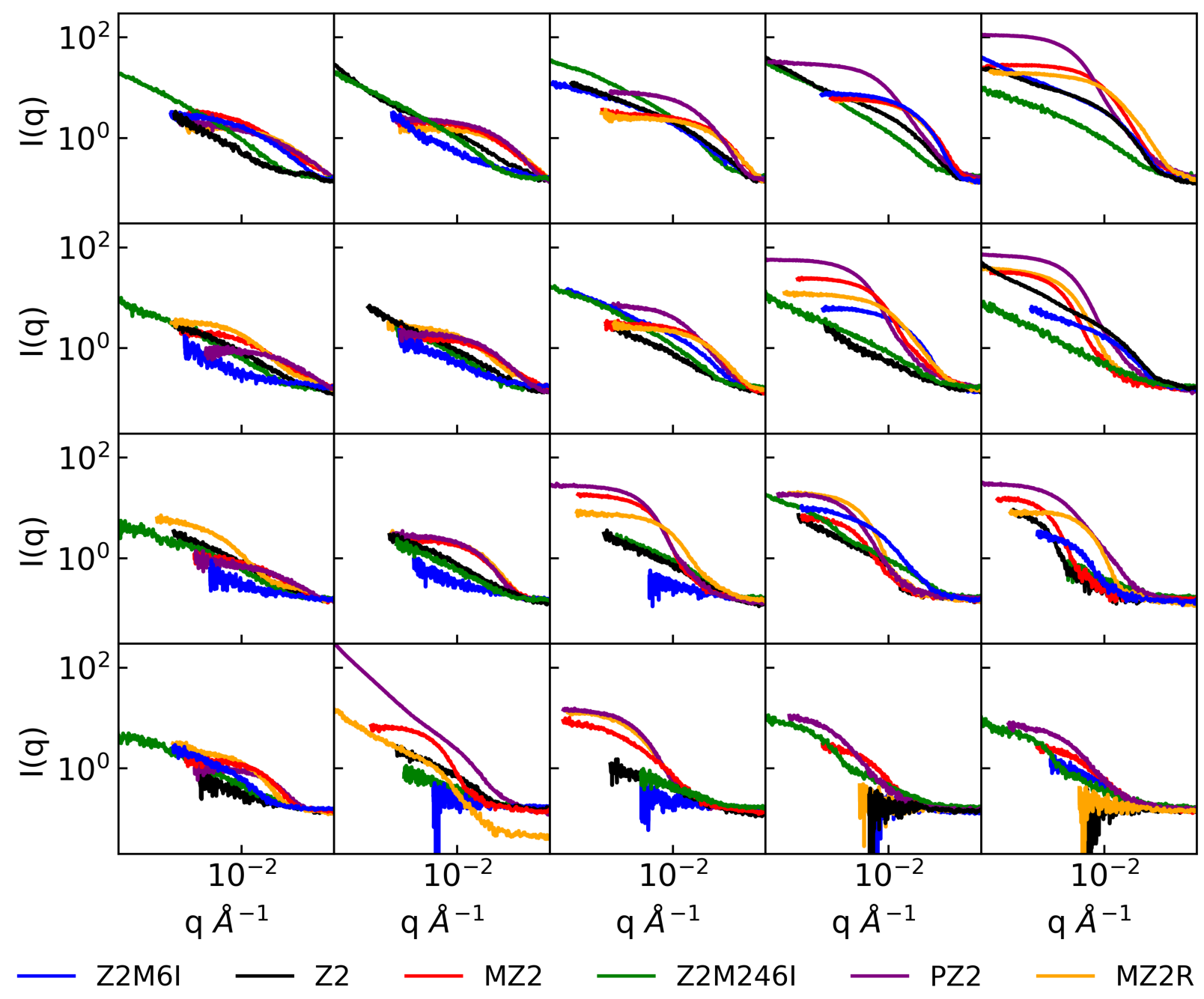

Figure 18: Smeared USAXS data. Sample compositions: $1 \mathrm{mM}$ HEPES; left to right 0.040, 0.060, 0.089, 0.134, and $0.200 \mathrm{mM}$; top to bottom $0.200,0.117,0.068$, and $0.040 \mathrm{mM}$.

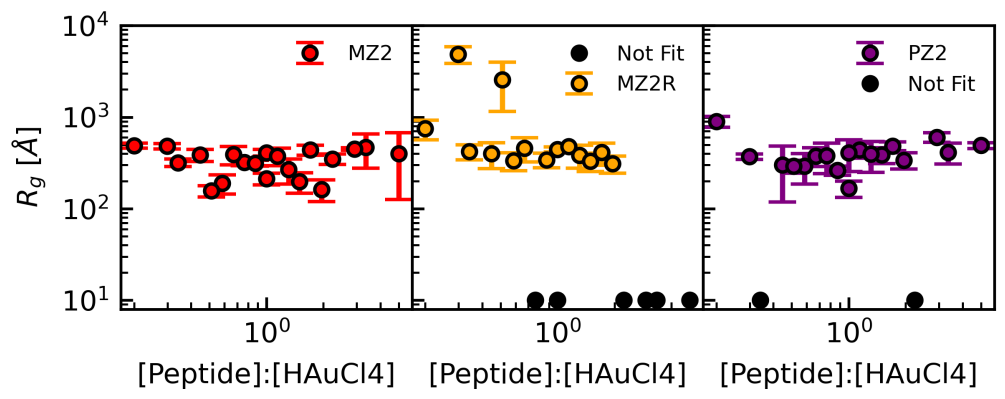

Figure 19: Radius of gyration results obtained from AUTORG plotted as a function of peptide: $\mathrm{HAuCl}_{4}$ molar ratio for all lipidated peptides and a HEPES concentration of $0 \mathrm{mM}$. Error bars indicate standard deviation. Profiles which did not contain a Guinier region that could be fitted were marked using black and plotted at a fixed $R_{g}$ of $10 \AA$. 


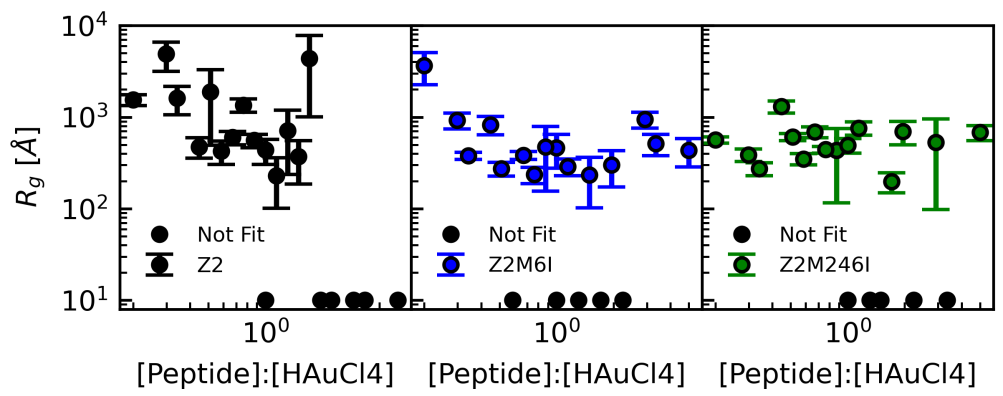

Figure 20: Radius of gyration results obtained from AUTORG plotted as a function of peptide: $\mathrm{HAuCl}_{4}$ molar ratio for non-lipidated peptides (except AG3) and a HEPES concentration of $0 \mathrm{mM}$. Error bars indicate standard deviation. Profiles which did not contain a Guinier region that could be fitted were marked using black and plotted at a fixed $R_{g}$ of $10 \AA$.

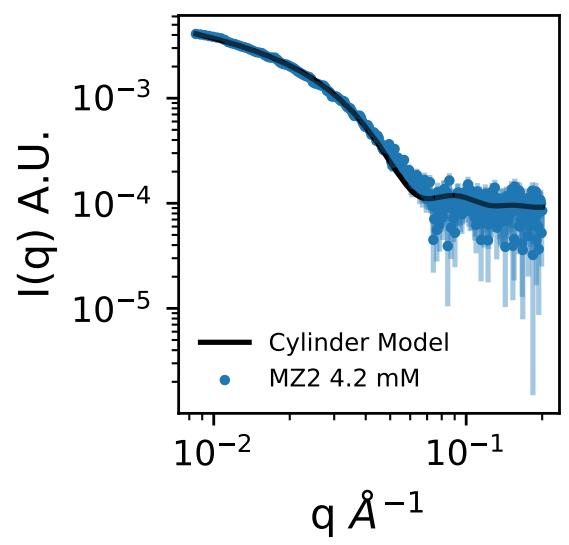

Figure 21: Smeared small-angle scattering of MZ2 dissolved in water $(4.2 \mathrm{mM})$ fit with a cylinder model (radius $=5.2$ $\mathrm{nm})$.

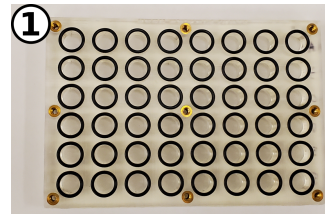

\section{(2)}

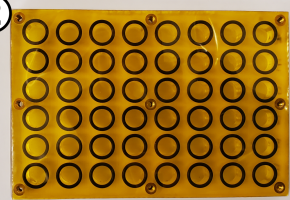

(3)

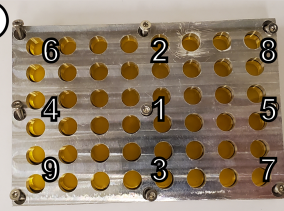

(4)

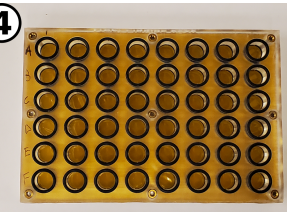

(5)

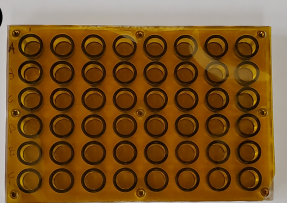

(6)

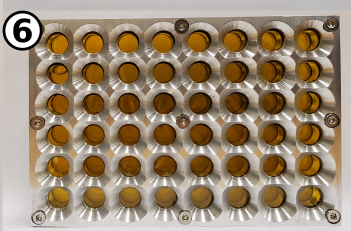

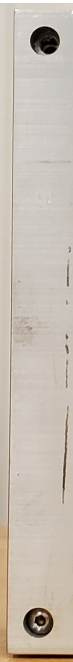

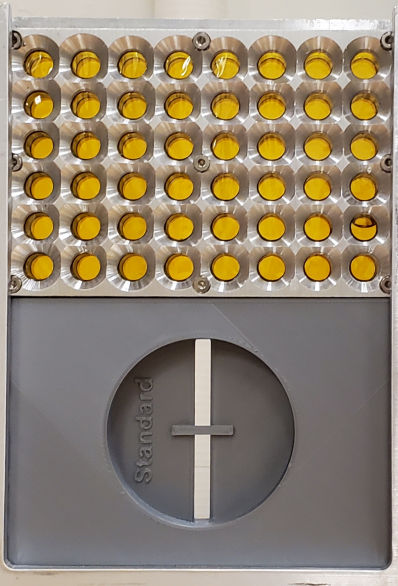

()

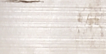

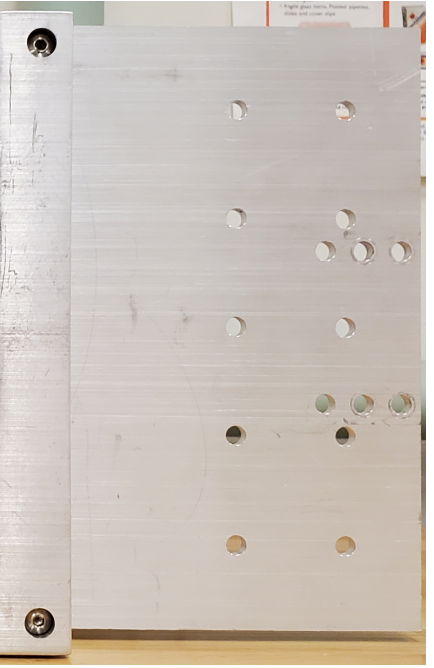

Figure 22: Process of assembling USXAXS sample holder, and the frame which holds up to two plates during experiments. A 3D printed mock cartridge is used to hold silver behenate for USAXS calibration. 


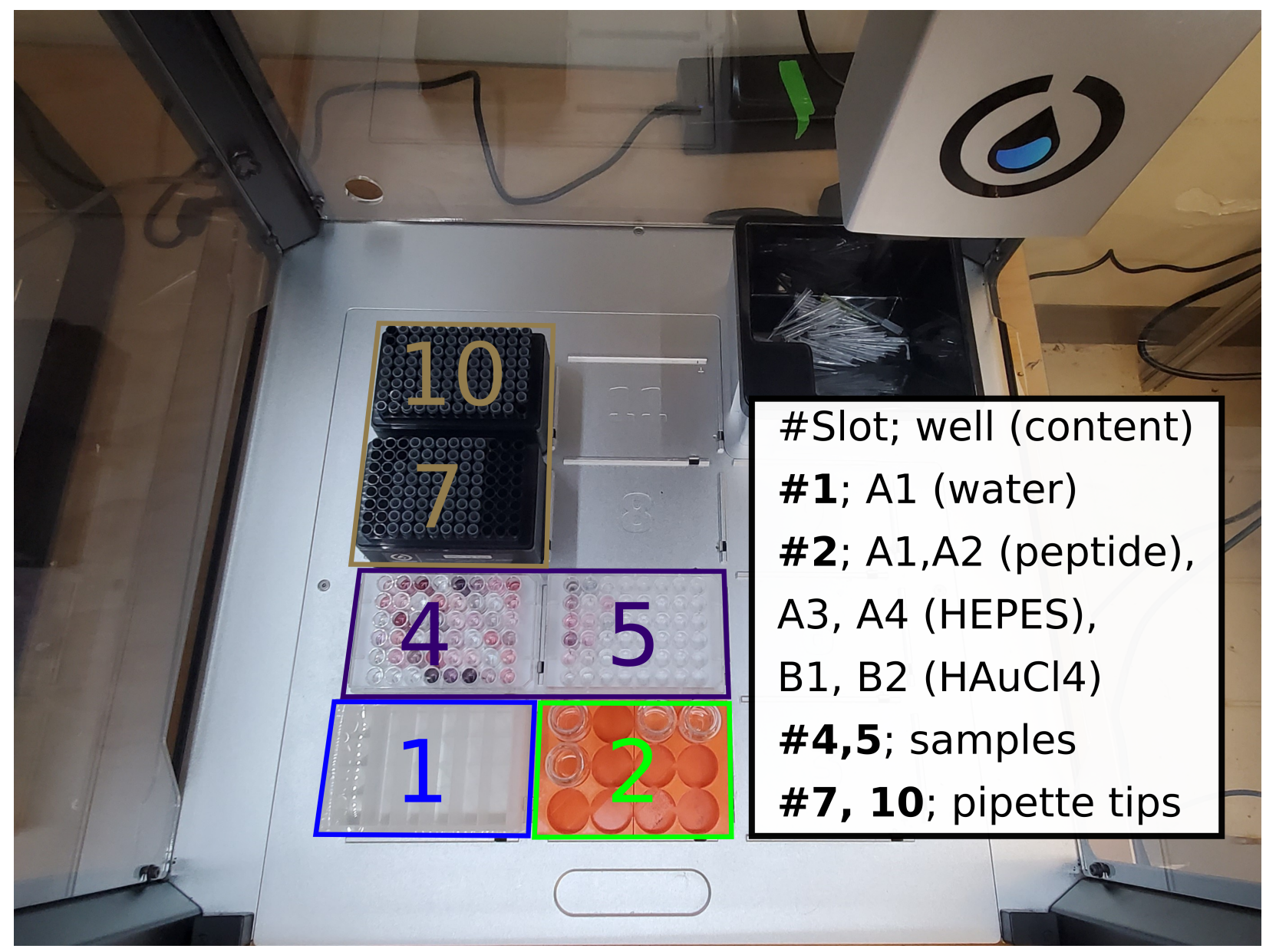

Figure 23: Opentrons OT-2 deck layout: 1, Agilent 6 column reservoir, water; 2, stocks in 20 mL scintillation vials seated in 3D printed vial holder; 4 and 5, 48 well microplates; 7 and 10, $300 \mu L$ pipette tips. Stock distribution in slot 2: A1, peptide; $\mathrm{A} 3$ and $\mathrm{A} 4$, HEPES; B1, $\mathrm{HAuCl}_{4}$ 


\section{References}

[1] Vincenzo Amendola, Roberto Pilot, Marco Frasconi, Onofrio M. Maragò, and Maria Antonia Iatì. Surface plasmon resonance in gold nanoparticles: A review. Journal of Physics Condensed Matter, 29(20), 2017.

[2] Erik C. Dreaden, Alaaldin M. Alkilany, Xiaohua Huang, Catherine J. Murphy, and Mostafa A. El-Sayed. The golden age: Gold nanoparticles for biomedicine. Chemical Society Reviews, 41(7):2740-2779, 2012.

[3] Nicholas M. Bedford, Zak E. Hughes, Zhenghua Tang, Yue Li, Beverly D. Briggs, Yang Ren, Mark T. Swihart, Valeri G. Petkov, Rajesh R. Naik, Marc R. Knecht, and Tiffany R. Walsh. Sequence-Dependent Structure/Function Relationships of Catalytic Peptide-Enabled Gold Nanoparticles Generated under Ambient Synthetic Conditions. Journal of the American Chemical Society, 138(2):540-548, 2016.

[4] Matthew R. Jones, Kyle D. Osberg, Robert J. Macfarlane, Mark R. Langille, and Chad A. Mirkin. Templated Techniques for the Synthesis and Assembly of Plasmonic Nanostructures. Chemical Reviews, 111(6):3736-3827, jun 2011.

[5] Zhiliang Li, Bin Cai, Wenchao Yang, and Chun Long Chen. Hierarchical Nanomaterials Assembled from Peptoids and Other Sequence-Defined Synthetic Polymers. Chemical Reviews, 121(22):14031-14087, 2021.

[6] Matthew B. Dickerson, Kenneth H. Sandhage, and Rajesh R. Naik. Protein- and peptide-directed syntheses of inorganic materials. Chemical Reviews, 108(11):4935-4978, 2008.

[7] Jos J. M. Lenders, Lukmaan A. Bawazer, David C. Green, Harshal R. Zope, Paul H. H. Bomans, Gijsbertus de With, Alexander Kros, Fiona C. Meldrum, and Nico A. J. M. Sommerdijk. Combinatorial Evolution of Biomimetic Magnetite Nanoparticles. Advanced Functional Materials, 27(10):1604863, mar 2017.

[8] Michelle A. Nguyen, Zak E. Hughes, Yang Liu, Yue Li, Mark T. Swihart, Marc R. Knecht, and Tiffany R. Walsh. Peptide-Mediated Growth and Dispersion of Au Nanoparticles in Water via Sequence Engineering. Journal of Physical Chemistry C, 122(21):11532-11542, 2018.

[9] Yen Nee Tan, Jim Yang Lee, and Daniel I.C. Wang. Uncovering the design rules for peptide synthesis of metal nanoparticles. Journal of the American Chemical Society, 132(16):5677-5686, 2010.

[10] Catherine J. Munro and Marc R. Knecht. Solution Effects on Peptide-Mediated Reduction and Stabilization of Au Nanoparticles. Langmuir, 33(48):13757-13765, 2017.

[11] Yue Li, Zhenghua Tang, Paras N. Prasad, Marc R. Knecht, and Mark T. Swihart. Peptide-mediated synthesis of gold nanoparticles: effects of peptide sequence and nature of binding on physicochemical properties. Nanoscale, 6(6):3165-3172, 2014.

[12] Chen Zhang, Chengyi Song, H. Christopher Fry, and Nathaniel L. Rosi. Peptide conjugates for directing the morphology and assembly of 1D nanoparticle superstructures. Chemistry - A European Journal, 20(4):941-945, 2014.

[13] Soumitra Mokashi-Punekar, Tiffany R. Walsh, and Nathaniel L. Rosi. Tuning the Structure and Chiroptical Properties of Gold Nanoparticle Single Helices via Peptide Sequence Variation. Journal of the American Chemical Society, 141(39):15710-15716, 2019.

[14] Soumitra Mokashi-Punekar, Sydney C. Brooks, Camera D. Hogan, and Nathaniel L. Rosi. Leveraging Peptide Sequence Modification to Promote Assembly of Chiral Helical Gold Nanoparticle Superstructures. Biochemistry, 60(13):1044-1049, 2021.

[15] Chun Long Chen, Peijun Zhang, and Nathaniel L. Rosi. A new peptide-based method for the design and synthesis of nanoparticle superstructures: Construction of highly ordered gold nanoparticle double helices. Journal of the American Chemical Society, 130(41):13555-13557, 2008.

[16] Andrea D. Merg, Joseph Slocik, Martin G. Blaber, George C. Schatz, Rajesh Naik, and Nathaniel L. Rosi. Adjusting the Metrics of 1-D Helical Gold Nanoparticle Superstructures Using Multivalent Peptide Conjugates. Langmuir, 31(34):9492-9501, 2015.

[17] Chengyi Song, Gongpu Zhao, Peijun Zhang, and Nathaniel L. Rosi. Expeditious synthesis and assembly of sub-100 nm hollow spherical gold nanoparticle superstructures. Journal of the American Chemical Society, 132(40):14033-14035, 2010.

[18] Huachen Tao, Tianyi Wu, Matteo Aldeghi, Tony C. Wu, Alán Aspuru-Guzik, and Eugenia Kumacheva. Nanoparticle synthesis assisted by machine learning. Nature Reviews Materials, 0123456789:in press, 2021.

[19] Jakob C. Dahl, Xingzhi Wang, Xiao Huang, Emory M. Chan, and A. Paul Alivisatos. Elucidating the Weakly Reversible Cs-Pb-Br Perovskite Nanocrystal Reaction Network with High-Throughput Maps and Transformations. Journal of the American Chemical Society, 142(27):11915-11926, 2020. 
[20] Beau R. Peelle, Eric M. Krauland, K. Dane Wittrup, and Angela M. Belcher. Design Criteria for Engineering Inorganic Material- Specific Peptides. Langmuir, 21(15):6929-6933, jul 2005.

[21] Rajesh R. Naik, Sarah J. Stringer, Gunjan Agarwal, Sharon E. Jones, and Morley O. Stone. Biomimetic synthesis and patterning of silver nanoparticles. Nature Materials, 1(3):169-172, nov 2002.

[22] Jan Ilavsky, Fan Zhang, Ross N. Andrews, Ivan Kuzmenko, Pete R. Jemian, Lyle E. Levine, and Andrew J. Allen. Development of combined microstructure and structure characterization facility for in situ and operando studies at the advanced photon source. Journal of Applied Crystallography, 51:867-882, 2018.

[23] J. A. Lake. An iterative method of slit-correcting small angle X-ray data. Acta Crystallographica, 23(2):191-194, 1967.

[24] Jan Ilavsky and Peter R. Jemian. Irena: tool suite for modeling and analysis of small-angle scattering. Journal of Applied Crystallography, 42(2):347-353, Apr 2009.

[25] Maxim V. Petoukhov, Peter V. Konarev, Alexey G. Kikhney, and Dmitri I. Svergun. ATSAS 2.1 - towards automated and web-supported small-angle scattering data analysis. Journal of Applied Crystallography, 40(s1):s223-s228, Apr 2007.

[26] Thomas Hendel, Maria Wuithschick, Frieder Kettemann, Alexander Birnbaum, Klaus Rademann, and Jörg Polte. In Situ Determination of Colloidal Gold Concentrations with UV-Vis Spectroscopy: Limitations and Perspectives. Analytical Chemistry, 86(22):11115-11124, nov 2014.

[27] Ahsan Habib, Masaaki Tabata, and Ying Guang Wu. Formation of gold nanoparticles by Good's Buffers. Bulletin of the Chemical Society of Japan, 78(2):262-269, 2005.

[28] Kangze Liu, Zhonglei He, James F. Curtin, Hugh J. Byrne, and Furong Tian. A novel, rapid, seedless, in situ synthesis method of shape and size controllable gold nanoparticles using phosphates. Scientific Reports, 9(1):1-13, 2019.

[29] Jianping Xie, Jim Yang Lee, and Daniel I.C. Wang. Seedless, surfactantless, high-yield synthesis of branched gold nanocrystals in HEPES buffer solution. Chemistry of Materials, 19(11):2823-2830, 2007.

[30] Wenjing Xi and Amanda J. Haes. Elucidation of HEPES Affinity to and Structure on Gold Nanostars. Journal of the American Chemical Society, 141(9):4034-4042, 2019.

[31] Po Jung Jimmy Huang, Jeffy Yang, Kellie Chong, Qianyi Ma, Miao Li, Fang Zhang, Woohyun J. Moon, Guomei Zhang, and Juewen Liu. Good's buffers have various affinities to gold nanoparticles regulating fluorescent and colorimetric DNA sensing. Chemical Science, 11(26):6795-6804, 2020.

[32] Zhenghua Tang, J. Pablo Palafox-Hernandez, Wing Cheung Law, Zak E. Hughes, Mark T. Swihart, Paras N. Prasad, Marc R. Knecht, and Tiffany R. Walsh. Biomolecular recognition principles for bionanocombinatorics: An integrated approach to elucidate enthalpic and entropic factors. ACS Nano, 7(11):9632-9646, 2013.

[33] Jiao Cao, Wendong Jia, Jinyue Zhang, Xiumei Xu, Shuanghong Yan, Yuqin Wang, Panke Zhang, Hong Yuan Chen, and Shuo Huang. Giant single molecule chemistry events observed from a tetrachloroaurate(III) embedded Mycobacterium smegmatis porin A nanopore. Nature Communications, 10(1), 2019.

[34] R. Grantham. Amino Acid Difference Formula to Help Explain Protein Evolution. Science, 185(4154):862-864, sep 1974.

[35] K. Kalyanasundaram and J. K. Thomas. Environmental Effects on Vibronic Band Intensities in Pyrene Monomer Fluorescence and Their Application in Studies of Micellar Systems. Journal of the American Chemical Society, 99(7):2039-2044, 1977.

[36] JO Ramsay and BW Silvermann. Functional data analysis. springer series in statistics, 1998.

[37] Anuj Srivastava and Eric P Klassen. Functional and shape data analysis, volume 1. Springer, 2016.

[38] Benjamin Ricaud, Pierre Borgnat, Nicolas Tremblay, Paulo Gonçalves, and Pierre Vandergheynst. Fourier could be a data scientist: From graph fourier transform to signal processing on graphs. Comptes Rendus Physique, 20(5):474-488, 2019.

[39] Aliaksei Sandryhaila and José MF Moura. Discrete signal processing on graphs: Graph fourier transform. In 2013 IEEE International Conference on Acoustics, Speech and Signal Processing, pages 6167-6170. IEEE, 2013.

[40] Jerome Friedman, Trevor Hastie, and Robert Tibshirani. The elements of statistical learning, volume 1. Springer series in statistics New York, 2001.

[41] Anthony Y. Fong, Lenson Pellouchoud, Malcolm Davidson, Richard C. Walroth, Carena Church, Ekaterina Tcareva, Liheng Wu, Kyle Peterson, Bryce Meredig, and Christopher J. Tassone. Utilization of machine learning to accelerate colloidal synthesis and discovery. Journal of Chemical Physics, 154(22), 2021. 
[42] M. Hussain Sangji, Hiroaki Sai, Stacey M. Chin, Sieun Ruth Lee, Ivan R Sasselli, Liam C. Palmer, and Samuel I. Stupp. Supramolecular Interactions and Morphology of Self-Assembling Peptide Amphiphile Nanostructures. Nano Letters, 21(14):6146-6155, 2021.

[43] Sunjie Ye, Andy P. Brown, Ashley C. Stammers, Neil H. Thomson, Jin Wen, Lucien Roach, Richard J. Bushby, Patricia Louise Coletta, Kevin Critchley, Simon D. Connell, Alexander F. Markham, Rik Brydson, and Stephen D. Evans. Sub-Nanometer Thick Gold Nanosheets as Highly Efficient Catalysts. Advanced Science, 6(21), 2019.

[44] Judea Pearl. Causal inference in statistics: An overview. Statistics surveys, 3:96-146, 2009.

[45] Karen Sachs, Omar Perez, Dana Pe'er, Douglas A Lauffenburger, and Garry P Nolan. Causal protein-signaling networks derived from multiparameter single-cell data. Science, 308(5721):523-529, 2005.

[46] Jessica A. Hutchinson, Ian W. Hamley, Juan Torras, Carlos Alemán, Jani Seitsonen, and Janne Ruokolainen. Self-Assembly of Lipopeptides Containing Short Peptide Fragments Derived from the Gastrointestinal Hormone PYY 3-36 : From Micelles to Amyloid Fibrils. The Journal of Physical Chemistry B, 123(3):614-621, jan 2019.

[47] Lucas Piñeiro, Mercedes Novo, and Wajih Al-Soufi. Fluorescence emission of pyrene in surfactant solutions. Advances in Colloid and Interface Science, 215:1-12, 2015.

[48] J Derek Tucker, Wei Wu, and Anuj Srivastava. Generative models for functional data using phase and amplitude separation. Computational Statistics \& Data Analysis, 61:50-66, 2013.

[49] Paul R Halmos. What does the spectral theorem say? The American Mathematical Monthly, 70(3):241-247, 1963.

[50] Lars Onsager. the Effects of Shape on the Interaction of Colloidal Particles. Annals of the New York Academy of Sciences, 51(4):627-659, 1949.

[51] Gérard Fournet. Étude théorique et expérimentale de la diffusion des rayons X par les ensembles denses de particules. Bulletin de la Société française de Minéralogie et de Cristallographie, 74(1):37-172, 1951.

[52] Jan Skov Pedersen. Analysis of small-angle scattering data from colloids and polymer solutions: Modeling and least-squares fitting. Advances in Colloid and Interface Science, 70(1-3):171-210, 1997.

[53] Lilo Pozzo, Kacper Lachowski, Sage Scheiwiller, and Edwin Antonio. pozzo-research-group/AutomationHardware: v0.1.0 - Initial release for documenting liquid sample holder on Zenodo, January 2022. 